\title{
The dynamical Matryoshka model: 1. Incoherent neutron scattering functions for lipid dynamics in bilayers
}

\author{
Dominique J. Bicout ${ }^{\mathrm{a}, \mathrm{d}, *}$, Aline Cisse ${ }^{\mathrm{b}, \mathrm{d}}$, Tatsuhito Matsuo ${ }^{\mathrm{b}, \mathrm{c}, \mathrm{d}}$, Judith Peters ${ }^{\mathrm{b}, \mathrm{d}, \mathrm{e}}$ \\ ${ }^{a}$ Univ. Grenoble Alpes, CNRS, Grenoble INP, VetAgro Sup, TIMC, 38000 Grenoble, France \\ ${ }^{b}$ Univ. Grenoble Alpes, CNRS, LiPhy, Grenoble, France \\ ${ }^{c}$ Institute for Quantum Life Science, NIQRST, Tokai, Ibaraki, 319-1106, Japan \\ ${ }^{d}$ Institut Laue-Langevin, 71 Avenue des Martyrs, 38042 Grenoble, France \\ ${ }^{e}$ Institut Universitaire de France, France
}

\begin{abstract}
Fluid lipid bilayers are the building blocks of biological membranes. Although there is a large amount of experimental data using inconsistent quasi-elastic neutron scattering (QENS) techniques to study membranes, very little theoretical works have been developed to study the local dynamics of membranes. The main objective of this work is to build a theoretical framework to study and describe the local dynamics of lipids and derive analytical expressions of inconsistent diffusion functions (ISF) for QENS. As results, we developed the dynamical Matryoshka model which describes the local dynamics of lipid molecules in membrane layers as a nested hierarchical convolution of three motional processes: (i) individual motions described by the vibrational motions of H-atoms; (ii) internal motions including movements of the lipid backbone, head groups and tails, and (iii) molecule movements of the lipid molecule as a whole. The analytical expressions of the ISF associated with these movements are all derived. For use in analyzing the QENS experimental data, we also derived an analytical expression for the aggregate ISF of the Matryoshka model which involves an elastic term plus three inelastic terms of well-separated time scales and whose amplitudes and rates are functions of the lipid motions. And as an illustrative application, we used the aggregated ISF to analyze the experimental QENS data on a lipid sample of multilamellar bilayers of DMPC (1,2-dimyristoyl-sn-glycero-3-phosphocholine). It is clear from this analysis that the dynamical Matryoshka model describes very well the experimental data and allow extracting the dynamical parameters of the studied system.
\end{abstract}

Keywords: lipids, bilayers, local dynamics, quasi-elastic neutron scattering, modeling

\section{Introduction}

Biological membranes are complex lipid-rich systems that constitute fundamental interfaces and selectively permeable barriers for the compartmentalization that defines cells and

\footnotetext{
* Corresponding author

Email address: bicout@ill.fr (Dominique J. Bicout)
} 
organisms. Biological membranes are composed of lipids, which self-assemble into bilayers, proteins and carbohydrates [1]. In addition to separating the interior from the exterior of cells, for example, membranes are very dynamic systems that host and ensure many essential processes vital for cellular functions, such as the transport of proteins or ions $[1,2]$. This dynamics is made up of both the activity at the surface and the movements of the bilayers which give the membrane an important fluid character to ensure its functions. Since lipids are the most abundant constituents of membranes, studies on the dynamics of lipids in membranes are and remain very crucial. Due to their complex structure and dynamics, lipid bilayers are characterized by a hierarchy and heterogeneity of motions over a wide range of time and space. These dynamics include, for example, local movements like lipid rotational, in-and-out of the plane diffusion at very short spatial scales and time scales of pico to nanoseconds, but also collective lipid movements like density fluctuations of short wavelength in pico to nanosecond range and long-wavelength flip-flop or undulation and bending modes of the bilayer in the nano to microsecond range $[2,3,4]$. In this work, we will only deal with short-range movements carried over short time scales (pico to nanoseconds).

Over the years, incoherent quasi-elastic neutron scattering (QENS) has proven to be a key technique for investigations of lipid motions at the pico - to nanosecond time scale and there is a great amount of experimental data that have been accumulated using QENS techniques $[5,6,7,8,9,10]$. However, since the seminal work by Pfeiffer et al. [5], there are very few theoretical works that have been developed to analyze and describe local dynamics of membranes [5, 11]. Along these lines, a study on phospholipid membranes has been proposed to separate the motions over three distinct time scales [12]. Using such models with QENS data allow to retrieve parameters like mean size of the solvent lipid cages, diffusion coefficients lipid rotations or in-plane Brownian motions. Motivated by these experimental investigations and findings, our main objective in this paper is to provide a theoretical framework of a model of membrane layers and to derive analytical expressions of incoherent structure functions (ISF) describing the local dynamics. To this end, we have developed a model that describes a membrane layer as a system of dynamically equivalent lipid molecules, each of which consisting of two connected (via a backbone) bodies (head and tail) undergoing kinds of internal and body motions.

The remaining of the paper is organized as follows. Section 2 consists of three parts: (i) formulation of the dynamical Matryoshka model which describes the hierarchical convolution of movements in the dynamics of a lipid molecule, (ii) detailed description of motions that are included in the model and derivations of analytical expressions of the associated ISFs, and (iii) derivation of an aggregate expression of the global ISF to be used to analyze and fit the experimental data. Technical details of all derivations are described in the Appendices A - C. Section 3 illustrates how the developed theory can be used to analyze the experimental QENS data of a bilayer membrane and extract the parameters of interest. Finally, the main results of the paper are summarized in the Section 4. 
bioRxiv preprint doi: https://doi.org/10.1101/2021.09.21.461198; this version posted September 24, 2021. The copyright holder for this preprint (which was not certified by peer review) is the author/funder, who has granted bioRxiv a license to display the preprint in perpetuity. It is made available under aCC-BY-NC-ND 4.0 International license.

\section{Model formulation and analytical expressions}

The membrane layer is considered as a system made of structurally and dynamically equivalent lipid molecules interacting with each other. Among the whole hierarchy of motions characterizing the membrane dynamics, we are interested in the local motions of lipid molecules as can be studied using techniques like incoherent quasi-elastic neutron scattering, inelastic x-ray scattering, to cite a few $[5,6,7,8,9$, 10]. Figure $1 \mathrm{~A}$ provides an illustration of the types of phospholipid molecules we will be dealing with. To describe the motional processes of an individual lipid molecule, occurring in the potential of mean force generated by the sea of lipid molecules in the membrane layer, we develop the Matryoshka model described below (see Fig. 1B).

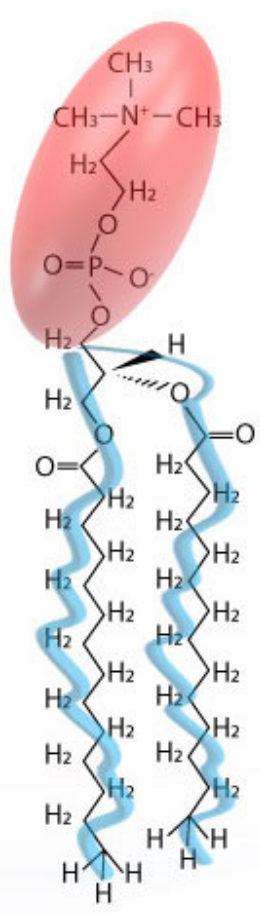

A: Lipid molecule

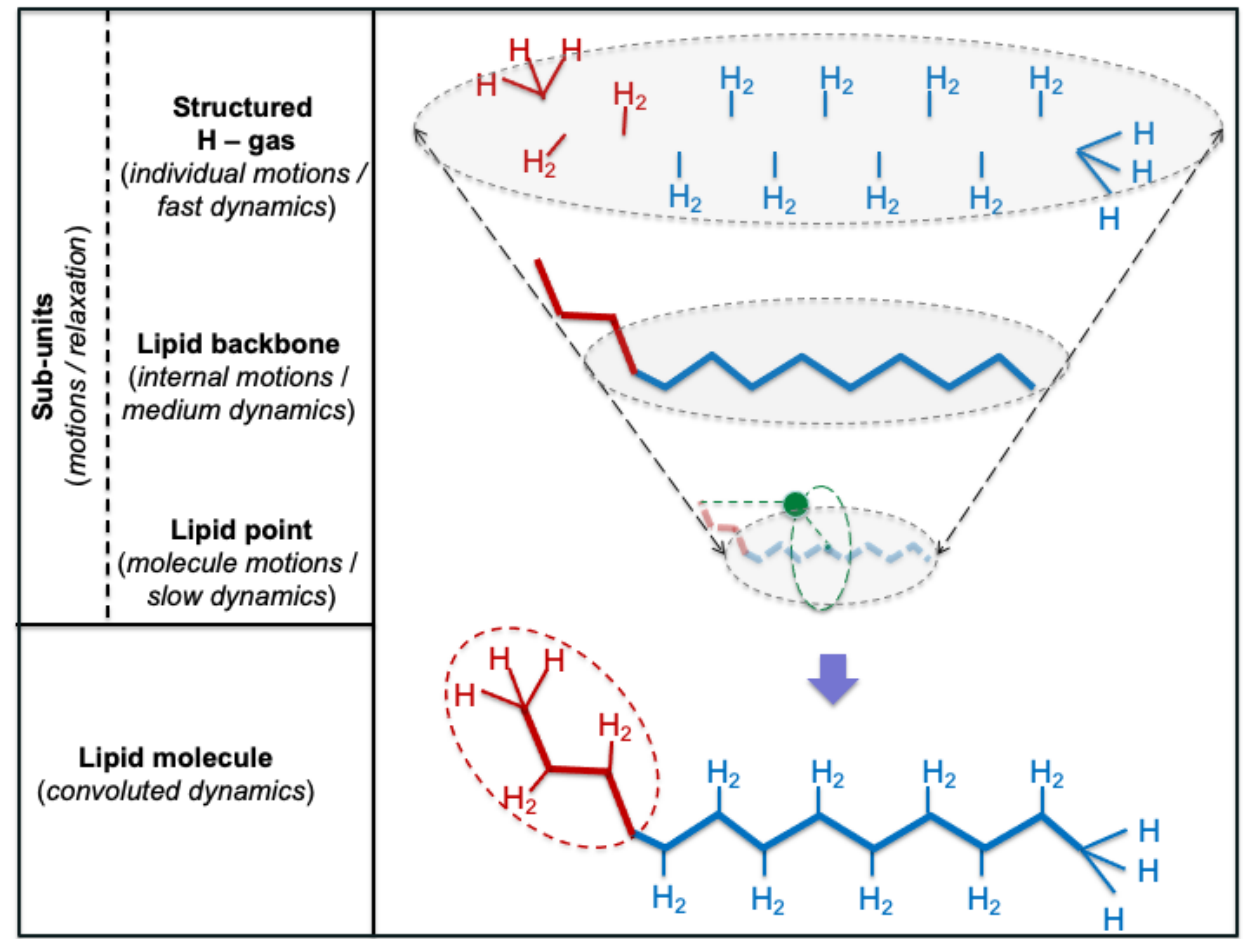

B: Dynamical Matryoshka model

Figure 1: A: Illustration of a phospholipid molecule 1,2-dimyristoyl-sn-glycero-3-phosphocholine (DMPC) consisting of a hydrophilic head (in red) and hydrophobic fatty acid tails (in blue) with the distribution of H-atoms. Courtesy from Steph Monfront (ILL). B: Dynamical Matryoshka model for the dynamics of the lipid molecule represented as a funnel of three - level convoluted dynamic processes. Lipid tails are represented by an effective single tail for the dynamics. Red and blue colored elements relate to head and tail groups, respectively. H-atoms with $\mathrm{C}-\mathrm{H}$ bounds (top) are structured along the lipid backbone (middle), with head and tail subunits, mirroring the lipid molecule representation (bottom of the figure). At the bottom of the funnel, the lipid molecule as a whole (rigid body) comes down to a point particle (green sphere) representing the center of inertia (barycenter) of all dynamic $\mathrm{H}$-atoms with respect to the lipid main axis (dashed lipid backbone). 


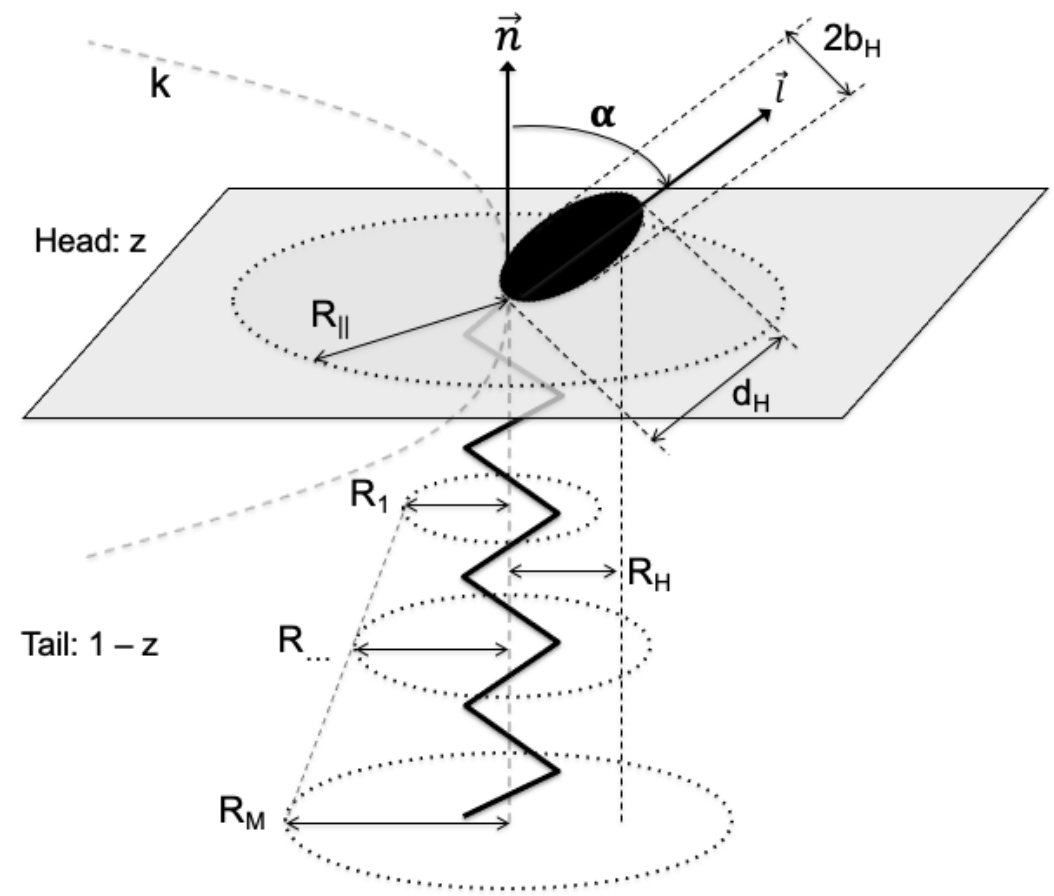

Figure 2: Scheme of a lipid molecule showing the characteristic parameters of motions. The polar head group is represented by the black ellipse (tilted by an angle $\alpha$ ) of short and long diameters $2 b_{\mathrm{H}}$ and $d_{\mathrm{H}}$, respectively, and the hydrophobic tails are represented by a dynamically effective tail (vertical zigzag black line) of apparent length $M$. Grey plane separates the head group (with a fraction $z$ of H-atoms) from the tail (with a fraction $1-z$ of H-atoms). The membrane normal (lipid axis) and head axis (tilted by an angle $\alpha$ ) are indicated by $\vec{n}$ and $\vec{l}$, respectively. Dashed circle of radius $R_{\|}$in the grey plane represents the effective cage for the in-plane diffusion and the dashed parabola of stiffness $k$, perpendicular to the membrane plane, indicates in-out of the plane motions of the lipid molecule. Dashed circles of increasing radii, $R_{1}, \cdots, R_{M}$, around the effective tail represent areas for the diffusion motions of Hs along the tails and $R_{\mathrm{H}}$ is the distance between the lipid axis $\vec{n}$ and the center of inertia of all H-atoms with respect to $\vec{n}$.

\subsection{Dynamical Matryoshka model}

As illustrated in Fig. 1B, the dynamical Matryoshka model describes the dynamics of a lipid molecule as resulting from the hierarchical convolution of three motional processes (from fastest to slowest motions): (i) individual motions of $\mathrm{H}$-atoms forming the cloud of structured $\mathrm{H}$-atoms bound to backbone atoms, (ii) internal motions of the lipid backbone or skeleton made up of non-H atoms and H-atoms bound to them, the head and tail subunits, and (iii) molecule motions or rigid body motions of the lipid molecule as a whole represented by the movements of the center of inertia all dynamic H-atoms with respect to the lipid main axis. The metaphor of dynamical Matryoshka (nesting dolls) originates from what these motional processes occur in a superimposed and nested way over various (and overlapping) timescales and, therefore, are resolved by zooming in or out over the associated characteristic timescales. 


\subsection{Dynamical Matryoshka model: Incoherent Structure Function (ISF)}

Within the framework of this model and the hypothesis of dynamic independence for different motions, the ISF for the local motions of a lipid molecule can be written as,

$$
I(Q, t)=I_{\text {ind }}(Q, t) \times I_{\text {int }}(Q, t) \times I_{\mathrm{mol}}(Q, t),
$$

where $I_{\text {ind }}(Q, t), I_{\text {int }}(Q, t)$ and $I_{\text {mol }}(Q, t)$ are the ISFs associated to individual, internal and molecular motions, respectively. In the following, we detail the motional processes of lipids included in the dynamical Matryoshka model with, whenever possible, the associated potentials of mean force, $V(\mathbf{r})$. The elastic incoherent structure factor (EISF) for each motion can be calculated as (see, Table 4),

$$
\operatorname{EISF}(Q)=\left|\frac{\int \mathrm{e}^{i \mathbf{Q} \cdot \mathbf{r}} \mathrm{e}^{-\beta V(\mathbf{r})} d \mathbf{r}}{\int \mathrm{e}^{-\beta V(\mathbf{r})} d \mathbf{r}}\right|^{2} ; \beta^{-1}=\mathrm{k}_{\mathrm{B}} \mathrm{T}, \text { thermal energy } .
$$

The relationships between all the dynamical processes are summarized in Table 1 with the details of derivations of IFSs provided in Appendix A and the analytical expressions of the ISFs reported in Table A.7.

Table 1: Dynamical processes

\begin{tabular}{|l|c|c|l|}
\hline \hline Dynamics & Subunit & Motions & Process \\
\hline Individual & H-atoms & vibrational Debye-Waller & Very Fast \\
\hline Internal & $\begin{array}{l}\text { C-H groups } \\
\text { (backbone) }\end{array}$ Tail & jump dynamics $\circledast \frac{\text { diffusion in circles }}{\text { rotational diffusion } \circledast \text { flip-flop }}$ & Fast \\
\hline \multirow{3}{*}{ Molecule } & Lipid Molecule & Intermediate \\
\cline { 3 - 4 } & & rotational diffusion & \\
& & in-out of the plane diffusion & Slow \\
& & 2d-diffusion in a cage & \\
\hline \hline
\end{tabular}

\subsubsection{Individual motions}

Individual motions relate to the vibrational motions of the cloud of $\mathrm{H}$-atoms bound to the lipid backbone atoms. Such motions are described by a harmonic potential of mean force, $V(r)=r^{2} / 2\left\langle u^{2}\right\rangle$, where $\left\langle u^{2}\right\rangle$ is the ensemble mean-square displacements of $\mathrm{H}$-atoms about their equilibrium positions. These individual movements being relatively fast $(\sim 100$ meV, [13]) for the timescales that concern us, the associated ISF is reduced to the EISF (i.e., time independent) and, in fact, is factored out in Eq.(1). The ISF for individual motions is therefore given by the Debye-Waller factor as, $I_{\text {ind }}(Q, t)=I_{\mathrm{DW}}(Q)=A_{\mathrm{DW}}(Q)$ (see Table A.7). 


\subsubsection{Internal motions}

Internal motions result from the combination of three motional processes from the lipid backbone, head and tail subunits. The headgroup and tails are considered as independent subunits each with $z$ and $1-z$ fraction of $\mathrm{H}$-atoms, respectively, and the lipid backbone common to both subunits includes all H-atoms (see Fig. 2). The ISF for internal motions writes as,

$$
I_{\text {int }}(Q, t)=I_{\mathrm{bkb}}(Q, t)\left[z I_{\text {head }}(Q, t)+(1-z) I_{\text {tail }}(Q, t)\right],
$$

where $I_{\mathrm{bkb}}(Q, t), I_{\text {head }}(Q, t)$ and $I_{\text {tail }}(Q, t)$ are the ISFs for motions in the lipid backbone, head and tail.

- ISF for the Backbone motions, $I_{\mathrm{bkb}}(Q, t)$ : Backbone motions relate to the motions of $\mathrm{C}-\mathrm{H}$ groups in the lipid molecule. Several models can be used to describe the heterogeneous dynamics of $\mathrm{C}-\mathrm{H}$ groups depending on whether they are located in the head group or in the tails. For simplicity, we considered that the movements of all the $\mathrm{C}-\mathrm{H}$ groups could be described using a jump dynamics between two non-equivalent sites distant from $d$ and characteristic relaxation rate $\Gamma_{\text {jump }}$. Because of heterogeneity, $d$ and $\Gamma_{\text {jump }}$ are ensemble averaged quantities over distributions of site distances and relaxation times, respectively. The potential of mean force for these motions is defined as [14],

$$
\frac{\mathrm{e}^{-\beta V(\mathbf{r})}}{\int \mathrm{e}^{-\beta V(\mathbf{r})} d \mathbf{r}}= \begin{cases}\phi & ; \quad \mathbf{r}=\mathbf{r}_{1} \\ 1-\phi & ; \quad \mathbf{r}=\mathbf{r}_{1}+d \\ 0 & ; \quad \text { otherwise }\end{cases}
$$

where $\mathbf{r}_{1}$ is the position of the low energy site and $\phi$ is the probability of occupying $\mathbf{r}_{1}$ (see Table A.7) and the ISF, $I_{\mathrm{bkb}}(Q, t)=I_{\text {jump }}(Q, t)$, is given in Table A.7.

- ISF for Tail motions, $I_{\text {tail }}(Q, t)$ : Tail motions refer to the movements of the H-atoms in all the tails. In Fig. 2, the tails, which interact with each other, are represented by a dynamically effective tail which indicates the axis of the tails and especially the surfaces within which the movements of the $\mathrm{H}$-atoms take place. The movements of $\mathrm{H}$-atoms in the tails are modeled by classical two-dimensional diffusions, all of an effective diffusion constant $D_{\text {tail }}$ within circles of radii $R_{m}$ in the plane parallel to the membrane at $m$, where $m$ designates the position of the carbon atoms $C-C$ along the lipid tail from the start to the tail end (with, $1 \leq m \leq M$ ) and $M$ is the apparent length of the lipid tail. As shown in Fig. 2, as a result of interactions between tails, $R_{m}$ increases with $m$ as the explorable space increases with $m$. Unlike the linear increase of Carpentier et al. [15], we use instead, $R_{m}=R_{1} \sqrt{m}$, to somehow account for a random walk-like (along the tail axis) positions of carbon atoms to which hydrogens are bound. The potential of mean force for these motions at each $m$ is,

$$
V(r, \theta)= \begin{cases}0 & ; \quad 0 \leq r \leq R_{m}, 0 \leq \theta \leq 2 \pi \\ \infty \quad ; \quad \text { otherwise }\end{cases}
$$

and the $\operatorname{ISF} I_{\text {tail }}(Q, t)$ is given in Table A.7. 
- ISF for Head motions, $I_{\text {head }}(Q, t)$ : Headgroup motions, involved in the roughness of the membrane surface, include three independent motions (see Fig. 2): the uniaxial rotational diffusion about the head axis $\vec{l}$, the flip-flop or jump dynamics of $\vec{l}$ axis between angles $-\alpha$ and $\alpha$ about the membrane normal $\vec{n}$ axis and the uniaxial rotational diffusion of the $\vec{l}$ axis about the membrane normal $\vec{n}$ axis. However, for our purpose, by symmetry the rotational diffusion about the $\vec{n}$ axis averages out such that only the rotation about $\vec{l}$ and the flip-flop about $\vec{n}$ are retained. The headgroup size $b_{\mathrm{H}}$ (see Fig. 2) represents the distance between the head axis and the center of inertia of all H-atoms of the head group with respect to the head axis $\vec{l}$. Derivation of the ISF $I_{\text {head }}(Q, t)$ is detailed in Appendix $\mathrm{A}$.

\subsubsection{ISF for molecule motions: $I_{\mathrm{mol}}(Q, t)$}

For molecule or rigid body movements, all dynamic H-atoms together perform the same processes regardless of their location in the lipid molecule. Formally, the dynamics of the lipid molecule as a whole can therefore be described by that of the center of inertia of all dynamic H-atoms with respect to the lipid main axis (Fig. 1B). Three types of independent movements are considered: rotational motions around the axis $\vec{n}$ of the lipid molecule, in-out of the membrane plane movements (parallel to $\vec{n}$ ) and in-plane lateral diffusion of the lipid molecule. The ISF for molecular motions writes as,

$$
I_{\text {mol }}(Q, t)=I_{\text {rot }}(Q, t) \times I_{\text {in-out }}(Q, t) \times I_{2 \mathrm{~d}}(Q, t),
$$

where $I_{\text {rot }}(Q, t), I_{\text {in-out }}(Q, t)$ and $I_{2 \mathrm{~d}}(Q, t)$ are the ISFs for rotational, in-out of the plane and in-plane diffusion motions.

- ISF for rotational motions, $I_{\text {rot }}(Q, t)$ : For the rotational motions of the lipid molecule about its axis $\vec{n}$, all $\mathrm{H}$-atoms perform exactly the same rotational movement about the lipid axis $\vec{n}$. However, as not all of the H-atoms are located equidistant from the lipid axis, the resulting ISF should be the sum of the individual ISFs. As a first approximation, we can reduce the rotational motions of all $\mathrm{H}$-atoms to that of their center of inertia and describe the rotational motions of the lipid molecule about its axis $\vec{n}$ by a rotational diffusion of diffusion coefficient $D_{\text {rot }}$ on a circle of radius $R_{\mathrm{H}}$, where $R_{\mathrm{H}}$ is the distance between the lipid axis and the center of inertia of all dynamic $\mathrm{H}$-atoms with respect to the lipid axis $\vec{n}$. The $\operatorname{ISF} I_{\text {rot }}(Q, t)$ is given in Table A.7.

- ISF for in-out of the plane motions, $I_{\text {in-out }}(Q, t)$ : The in-out-of-plane movements, involved in the roughness of the membrane surface, relate on the up and down motions (normal to the membrane plane) around the equilibrium position of the lipid molecule within the membrane layer. Such motions can be described by the one-dimensional (parallel to $\vec{n}$ ) diffusion within a harmonic potential of force constant $k$ and relaxation time $\tau$. The harmonic potential of mean force is given by, $V(z)=k z^{2} / 2$, where $z$ is the lipid molecule position around its equilibrium position. The $\operatorname{ISF} I_{\text {in-out }}(Q, t)$ is given in Table A.7. 
- ISF for in-plane 2d diffusion motions, $I_{2 \mathrm{~d}}(Q, t)$ : In general, in-plane lateral diffusion of lipid molecules is composed of short range local diffusion in a solvent cage and long range jumps between different sites allowing molecules to travel within the membrane layer. As this work is dealing with spatially short range dynamics, we will only consider short range diffusion in what follows. Thus, in-plane diffusion of lipid molecules describes when molecules exchange places via Brownian motion within a cage. Such motions are described by a two-dimensional diffusion of diffusion constant $D_{\|}$within a circle of radius $R_{\|}$, where $R_{\|}$is the effective size of the cage formed by the neighboring lipid molecules. The potential of mean force for such a confined isotropic two-dimensional diffusion is given by,

$$
V(r, \theta)= \begin{cases}0 & ; \quad 0 \leq r \leq R_{\|}, \forall \theta \\ \infty \quad ; \quad \text { otherwise }\end{cases}
$$

where $r$ is the position of lipid in the membrane plane. The $\operatorname{ISF} I_{2 \mathrm{~d}}(Q, t)$ is given in Table A.7.

All the 7 movements included in the dynamical Matryoshka model and associated 18 dynamical parameters are summarized in the Table 2.

Table 2: Local motions and associated parameters of the Matryoshka model.

\begin{tabular}{|c|c|c|c|}
\hline Dynamics & Motions & Parameter & Parameter definition \\
\hline Individual & Vibrational Debye-Waller & $\left\langle u^{2}\right\rangle$ & H's mean-square displacements \\
\hline \multirow{11}{*}{ Internal } & \multirow{3}{*}{ Backbone: jump dynamics } & $d$ & two-site distance \\
\hline & & $\phi$ & probability of jump events \\
\hline & & $\Gamma_{\text {jump }}$ & jump relaxation rate \\
\hline & \multirow{4}{*}{ Tails: diffusion in circles } & $1-z$ & fraction of H's in the tail \\
\hline & & $M$ & length of the effective tail \\
\hline & & $R_{1}$ & radius of the first circle \\
\hline & & $D_{\text {tail }}$ & diffusion coefficient of tail H's \\
\hline & \multirow{4}{*}{ Head: rotational diffusion + filp-flop } & $\alpha$ & head tilt angle \\
\hline & & $b_{\mathrm{H}}$ & headgroup H-radius \\
\hline & & $\tau_{\mathrm{ff}}$ & inter flip-flop mean resting time \\
\hline & & $D_{\text {head }}$ & rotational diffusion coefficient \\
\hline \multirow{6}{*}{ Molecule } & \multirow{2}{*}{ Rotational diffusion } & $R_{\mathrm{H}}$ & H-radius of the lipid molecule \\
\hline & & $D_{\text {rot }}$ & rotational diffusion coefficient \\
\hline & \multirow{2}{*}{ In-out of the plane diffusion } & $k$ & force constant \\
\hline & & $\tau$ & relaxation time \\
\hline & \multirow{2}{*}{ 2d-diffusion in a cage } & $R_{\|}$ & lipid cage radius \\
\hline & & $D_{\|}$ & molecular diffusion coefficient \\
\hline
\end{tabular}

\subsection{Aggregated processes: 3-dynamical process approximation}

As outlined above, the ISF of local movements of lipid molecules results from the convolution of several individual ISFs which, in general, are multi-exponential functions of time. 
Even when each individual ISF was approximated by a single exponential relaxation, the resulting ISF would still be multi-exponential. In practice, it would be challenging to use such a multi-exponential function in the analyzes of experimental data to extract physical parameters of interest listed in Table 2. The idea is therefore to develop an approximation of the ISF allowing to reduce timescales by coupling them together while keeping the original information (as summarized in Table 2). For that purpose, we follow Wanderlingh et al. [12] and consider how the dynamics of the lipid molecules as described above can be approximatively aggregated into 3-dynamical processes. Under the context of dynamical processes taking place on widely separated time scales (one or few orders of magnitude) as outlined above, the main value of the 3-dynamical process model is to allow fitting the experimental data and, therefore, providing an opportunity for direct connection between analytical models of lipid motions and experimental observations.

The 3-dynamical process model is a phenomenological model in which the position $\mathbf{r}(t)$ at time $t$ of the $\mathrm{H}$-atom in lipid molecules can be split into three independent components, $\mathbf{r}(t)=\mathbf{r}_{1}(t)+\mathbf{r}_{2}(t)+\mathbf{r}_{3}(t)$, where $\mathbf{r}_{1}(t), \mathbf{r}_{2}(t)$ and $\mathbf{r}_{2}(t)$ stand for the slow, intermediate and fast motions, respectively, describing the overall motions of hydrogen atoms in the equilibrium lipid molecule at very different time scales. Each of these motions accounts for a confined dynamics with a quasi-elastic term described by a single exponential decay term. Under the approximation of separate time scales, the aggregated ISF can be written as,

$$
I_{\text {agg }}(Q, t)=A_{\mathrm{DW}}(Q)\left[B_{0}+B_{1} \mathrm{e}^{-\Gamma_{1} t}+B_{2} \mathrm{e}^{-\Gamma_{2} t}+B_{3} \mathrm{e}^{-\Gamma_{3} t}\right] ; \Gamma_{0}=0<\Gamma_{1}<\cdots<\Gamma_{3},
$$

where $A_{\mathrm{DW}}(Q)$ stands for the Debye-Waller factor, $B_{0}$ is the overall EISF, $B_{i}$ and $\Gamma_{i}$ for $i=1,2,3$ are the amplitudes and relaxation rates of the slow, intermediate and fast motions, respectively, such that, $\sum_{i=0}^{3} B_{i}(Q)=1$ for all $Q$. Accordingly, the measurable function in incoherent neutron scattering experiments is,

$$
\begin{aligned}
S(Q, \omega) & =\mathcal{R}(Q, \omega) \circledast\left\{\frac{1}{\pi} \int_{0}^{+\infty} d t I_{\mathrm{agg}}(Q, t) \cos (\omega t)\right\} \\
& =\mathcal{R}(Q, \omega) \circledast\left\{A_{\mathrm{DW}}(Q) \sum_{i=0}^{3} B_{i}(Q) \mathcal{L}_{i}(Q, \omega)\right\} ; \mathcal{L}_{i}\left(\Gamma_{i} ; Q, \omega\right)=\frac{1}{\pi} \frac{\Gamma_{i}(Q)}{\omega^{2}+\Gamma_{i}^{2}(Q)},(9)
\end{aligned}
$$

where $\mathcal{R}(Q, \omega)$ refers to the experimental resolution function, $B_{i}(Q)$ and $\Gamma_{i}(Q)$ are the respective areas and half-widths at half-maximum (HWHM) of the Lorentzian functions $\mathcal{L}_{n}(Q, \omega)$. It should be stressed here that:

(i) the three time scales slow, intermediate and fast do not necessarily correspond or coincide to and, therefore, are not to be confused with those of individual, internal and collective motions (see Table 1);

(ii) the phenomenological model does not provide which movements are resolved at each timescale. 
The purpose of aggregating the $I(Q, t)$, derived above and given in Eq.(1), into 3-dynamical processes is to associate each term in Eq.(8) with a physical meaning and an analytical expression. To this end, we use the mapping, $I(Q, t) \approx I_{\text {agg }}(Q, t)$, i.e.,

$$
\begin{aligned}
& I_{\text {jump }}(Q, t)\left[z I_{\text {head }}(Q, t)+(1-z) I_{\text {tail }}(Q, t)\right] I_{\text {rot }}(Q, t) I_{\text {in }- \text { out }}(Q, t) I_{2 \mathrm{~d}}(Q, t) \\
& \approx B_{0}+B_{1} \mathrm{e}^{-\Gamma_{1} t}+B_{2} \mathrm{e}^{-\Gamma_{2} t}+B_{3} \mathrm{e}^{-\Gamma_{3} t},
\end{aligned}
$$

to derive the expressions of $B$ 's and $\Gamma$ 's as functions of physical quantities in $I(Q, t)$. Note that in Eq.(10), $B_{0}$ is already known and is equal to $B_{0}=I(Q, t \rightarrow \infty)=I_{\text {agg }}(Q, t \rightarrow$ $\infty)$ (i.e., the product of EISF of all local motions), whereas the $B_{i>0}$ and $\Gamma_{i>0}$ remain to be determined. In addition, the number of terms after the approximate sign " $\approx$ " in Eq.(10) can be reduced when some of the amplitudes cancel out because the contributing motions turn out to be not observable. The aggregation procedure, based on the hierarchy of relaxation time scales of motional processes in the Matryoshka model, is detailed in Appendix B and the results are summarized in Table 3 in which the expressions of original EISFs and relaxation rates are given in Tables 4 and 5, respectively. Table 3 provides which local motions contribute at each timescale of the 3-dynamical process and how they are combined in the expressions of amplitudes and relaxation rates. Each relaxation rate is not exclusive of a single local motion and the same local motion can therefore contribute to different timescales. The amplitudes result from the combination of several local motions whose relaxation times can be relatively different. From the amplitudes (with the DebyeWaller factoring all), we have the following contributions:

- Slow motions: backbone, head, tails, rotational, in-out of the plane and in-plane diffusion;

- Intermediate motions: backbone, head, tails and rotational;

- Fast motions: backbone and tails.

And, as a function of $Q$, we have:

- $Q \rightarrow 0$ limit: as expected, the scattering function essentially consists of the elastic peak (EISF) with all motions contributing and no Lorentzian functions as, $B_{0}(Q \rightarrow 0) \rightarrow 1$ and $B_{i>0}(Q \rightarrow 0) \rightarrow 0$

- Moderate $Q$ : as all $B_{i} \neq 0$, the scattering function consists of the elastic peak plus the three Lorentzian functions with contributions of all motions. Note that slow motions are only observable for these $Q$-ranges as $B_{1}(Q) \rightarrow 0$ in both $Q \rightarrow 0$ and large $Q$ limits;

- Large $Q$ limit: as $B_{i \leq 1}(\operatorname{large} Q) \rightarrow 0$ and $B_{2}(\operatorname{large} Q)=1-B_{3}(\operatorname{large} Q) \approx z A_{\mathrm{bkb}}(\operatorname{large} Q) \neq$ 0 , the scattering function reduces to only two Lorentzian functions involving intermediate and fast motions and concerning motions of $\mathrm{C}-\mathrm{H}$ groups of the backbone. 
Table 3: Amplitudes (areas) and relaxation rates of the 3-dynamical process approximation. $I_{\text {agg }}(Q, t)=A_{\mathrm{DW}}(Q)\left[B_{0}+B_{1} \mathrm{e}^{-\Gamma_{1} t}+B_{2} \mathrm{e}^{-\Gamma_{2} t}+B_{3} \mathrm{e}^{-\Gamma_{3} t}\right]$

\begin{tabular}{|c|c|c|}
\hline \multirow{2}{*}{ Motions } & \multicolumn{2}{|l|}{ Amplitudes (areas) } \\
\hline & theoretical $^{(a)}$ & experimental $^{(b)}$ \\
\hline $\begin{array}{l}\text { EISF } \\
\text { Slow } \\
\text { Intermediate } \\
\text { Fast }\end{array}$ & $\begin{array}{l}B_{0}=\left[z A_{\text {head }}+(1-z) A_{\text {tail }}\right] A_{\text {jump }} A_{\text {rot }} A_{\text {in }- \text { out }} A_{2 \mathrm{~d}} \\
B_{1}=\left[z A_{\text {head }}+(1-z) A_{\text {tail }}\right]\left(1-A_{\text {in-out }} A_{2 \mathrm{~d}}\right) A_{\text {jump }} A_{\text {rot }} \\
B_{2}=A_{\text {jump }}\left[z\left(1-A_{\text {head }} A_{\text {rot }}\right)+(1-z) A_{\text {tail }}\left(1-A_{\text {rot }}\right)\right] \\
B_{3}=z\left(1-A_{\text {jump }}\right)+(1-z)\left(1-A_{\text {jump }} A_{\text {tail }}\right)\end{array}$ & $\begin{array}{l}A_{0}=m B_{0}+\varepsilon_{0} \\
\left\{A_{i}=m B_{i}+\frac{\left[1-m-\varepsilon_{0}\right]}{3}\right.\end{array}$ \\
\hline & Relaxation rates & \\
\hline Slow & $\Gamma_{1}=\frac{\left(1-A_{\text {in-out }}\right) \Gamma_{\text {in-out }}+\left(1-A_{2 \mathrm{~d}}\right) \Gamma_{2 \mathrm{~d}}}{1-A_{\text {in-out }} A_{2 \mathrm{~d}}}$ & \\
\hline Intermediate & $\Gamma_{2}=\frac{z\left(1-A_{\text {head }}\right) \Gamma_{\text {head }}+\left[z+(1-z) A_{\text {tail }}\right]\left(1-A_{\text {rot }}\right) \Gamma_{\text {rot }}}{z\left(1-A_{\text {head }} A_{\text {rot }}\right)+(1-z) A_{\text {tail }}\left(1-A_{\text {rot }}\right)}$ & \\
\hline Fast & $\Gamma_{3}=\frac{\left(1-A_{\text {jump }}\right) \Gamma_{\text {jump }}+(1-z)\left(1-A_{\text {tail }}\right) \Gamma_{\text {tail }}}{z\left(1-A_{\text {jump }}\right)+(1-z)\left(1-A_{\text {jump }} A_{\text {tail }}\right)}$ & \\
\hline
\end{tabular}

$\overline{(a)}$ Limiting properties for $B_{i}: B_{0}(Q \rightarrow 0)=1$ and $B_{i>0}(Q \rightarrow 0)=0 ; B_{i}($ high $Q) \rightarrow 0$ for both $i=0$ and $i=1$ while $B_{2}(\operatorname{large} Q)=1-B_{3}(\operatorname{large} Q)=z A_{\text {jump }}(\operatorname{large} Q)$ where $A_{\text {jump }}($ large $Q)=A_{\text {bkb }}($ large $Q) \approx$ $1-2 \phi(1-\phi)$.

(b) See Appendix $\mathrm{C}$ for derivations. $m$ is the fraction of dynamical or mobile H-atoms and the error function $\varepsilon_{0}(Q)$ accounts for immobile H-atoms, multiple scattering effects observable at low-Q, and others. For simplicity, we will assume that the errors are homogeneously distributed over length scales and, therefore, $\varepsilon_{0}(Q)=\varepsilon_{0}$. 


\section{Table 4: Elastic Incoherent Structure Factor (EISF) of dynamical processes.}

\begin{tabular}{|c|c|c|}
\hline Subunit & EISF & (a) $(\|, \perp)$ \\
\hline H-atom & $\begin{array}{l}\text { vibrational motions: Debye-Waller factor } \\
A_{\mathrm{DW}}(Q)=\exp \left\{-\frac{Q^{2}\left\langle u^{2}\right\rangle}{3}\right\}\end{array}$ & $(Y, Y)$ \\
\hline C-H groups & $\begin{array}{l}\text { jump dynamics between } 2 \text { non-equivalent sites distant from } d \\
A_{\text {jump }}(Q)=1-2 \phi(1-\phi)\left[1-j_{0}(Q d)\right]\end{array}$ & $(Y, Y)$ \\
\hline Tail & $\begin{array}{l}\text { 2d-diffusion of tail H-atoms inside } F(m) \text { - distribution of circles of radius } R_{m} \\
\text { (b) } A_{\text {tail }}(Q)=\sum_{m=1}^{M} F(m)\left[\frac{2 J_{1}\left(Q R_{m} \sin \gamma\right)}{Q R_{m} \sin \gamma}\right]^{2}\end{array}$ & $(Y, N)$ \\
\hline Head & $\begin{array}{l}\text { rotational diffusion of the headgroup about its axis plus flip-flop motion } \\
\text { (c) } A_{\text {head }}(Q)=\left[\sum_{l=-\infty}^{+\infty} J_{2 l}\left(Q b_{\mathrm{H}} \cos \gamma \sin \alpha\right) J_{l}\left(Q b_{\mathrm{H}} \sin \gamma \cos ^{2} \frac{\alpha}{2}\right) J_{l}\left(Q b_{\mathrm{H}} \sin \gamma \sin ^{2} \frac{\alpha}{2}\right)\right]\end{array}$ & $(Y, Y)$ \\
\hline \multirow{3}{*}{ Molecule } & $\begin{array}{l}\text { rotational diffusion of the lipid molecule about the membrane normal axis } \\
A_{\mathrm{rot}}(Q)=J_{0}^{2}\left(Q R_{\mathrm{H}} \sin \gamma\right)\end{array}$ & $(Y, N)$ \\
\hline & $\begin{array}{l}\text { in-out of the plane 1d-diffusion of the lipid molecule in a harmonic potential } \\
A_{\text {in-out }}(Q)=\exp \left\{-(Q \cos \gamma)^{2}\left(\frac{k_{\mathrm{B}} T}{k}\right)\right\}\end{array}$ & $(N, Y)$ \\
\hline & $\begin{array}{l}\text { in-plane 2d-diffusion of the lipid molecule inside a circle of radius } R_{\|} \\
A_{2 \mathrm{~d}}(Q)=\left[\frac{2 J_{1}\left(Q R_{\|} \sin \gamma\right)}{Q R_{\| \mid} \sin \gamma}\right]^{2}\end{array}$ & $(Y, N)$ \\
\hline
\end{tabular}

(a) $\mathbf{Q}$ direction: $\perp \Leftrightarrow \gamma=0$ and $\| \Leftrightarrow \gamma=\frac{\pi}{2}$ refer to $\mathbf{Q}$ directions perpendicular and parallel to the membrane plane, respectively. "Y=Yes" (i.e., $A_{i}(Q)$ changes with $Q$ ) and "N=No" (i.e., $A_{i}(Q)=1$ for all $Q$ ) indicate observable and non-observable motions, respectively. Limiting properties: as we are dealing with spatially restricted motions, $A_{\alpha}(Q=0)=1$ and $A_{\alpha}$ (high $\left.Q\right) \rightarrow 0$ for all continuous motions and for the jump process between small number of sites for (backbone) $A_{\text {jump }}($ large $Q) \neq 0$ and oscillates around, $1-2 \phi(1-\phi)$.

(b) For simplicity, we use $F(m)=1 / M$ and $R_{m}=R_{1} \sqrt{m}$.

(c) We have: $A_{\text {head }, \perp}(Q)=J_{0}^{2}\left(Q b_{\mathrm{H}} \sin \alpha\right)$ and $A_{\text {head,\|l }}(Q)=J_{0}^{2}\left(Q b_{\mathrm{H}} \cos ^{2} \frac{\alpha}{2}\right) J_{0}^{2}\left(Q b_{\mathrm{H}} \sin ^{2} \frac{\alpha}{2}\right)$ 


\section{Illustrative Example}

To compare and test how the theoretical developments outlined above would work when analyzing experimental data, we considered the following neutron scattering experiments. The whole task consists in determining 18 parameters describing the 7 motions of $\mathrm{H}$-atoms in the Matryoshka model (see, Table 2) plus 2 experimental parameters ( $m$ and $\varepsilon_{0}$ in Table $3)$.

\subsection{Quasi-Elastic Neutron Scattering (QENS) experiments and analyses}

We used lipid samples of multilamellar bilayers (MLBs) of DMPC (1,2-dimyristoyl-snglycero-3-phosphocholine) represented in Fig. 1A. The DMPC was purchased from Lipoid (Ludwigshafen Germany) or from Avanti Polar Lipids (Alabaster, USA) and used without further purification. The lipid samples were prepared on $6 \mathrm{Si}$ wafers, and hydrated in $\mathrm{D}_{2} \mathrm{O}$ atmosphere within a desiccator at full hydration. They were mounted on flat Aluminum sample holders, gold-coated to avoid sample contamination. The sealing of the cells was done by using Indium wire, and they were weighed before and after the experiment to check for any sample loss.

DMPC samples were scanned on the IN6 time-of-flight spectrometer from ILL (Grenoble, France), with a wavelength of $5.1 \AA$, corresponding to an energy resolution of about $70 \mu \mathrm{eV}$ [17]. At this resolution, motions up to around 10 ps are accessible and the attainable $Q$-range is of, $0.37 \leq Q \leq 2.02 \AA^{-1}$. The sample holder was oriented at $135^{\circ}$ from the beam to access in-plane motions [8]. QENS scans were performed at three different temperatures, $280 \mathrm{~K}$, $311 \mathrm{~K}$ and $340 \mathrm{~K}$, to probe the dynamics below and above the main phase transition of lipid bilayers; DMPC is known to undergo consecutive phase transitions from the gel phase to the ripple phase around $287 \mathrm{~K}$ and to the fluid phase around $297 \mathrm{~K}$ (corresponding to the physiological state in cells) [18].

Empty cell with and without wafers, as well as Vanadium, were measured for correction and normalization purposes. Raw data were first corrected by the empty cell +6 wafers contribution, using the Large Array Manipulation Program (LAMP) [19]. The resulting $\mathrm{S}(Q, \omega)$ spectra were subsequently analyzed in the range of $-10 \mathrm{meV} \leq \Delta \mathrm{E} \leq 2 \mathrm{meV}$ using IGOR Pro software (WaveMetrics, Lake Oswego, OR, USA). Following the QENS analysis in [12], the model used to fit the spectra is similar to that in Eq.(9) as,

$$
\mathrm{S}(Q, \omega)=A_{\mathrm{DW}}(Q)\left[\sum_{i=0}^{3} A_{i}(Q) \mathcal{L}_{i}(Q, \omega)\right] \circledast \mathcal{R}(Q, \omega)+\mathcal{B}(Q),
$$

where $A_{0}(Q)$ is the experimental elastic incoherent structure factor (EISF) and the $A_{i}(Q)$ for $i>0$ are the experimental areas of the Lorentzian functions $\mathcal{L}_{i}(Q, \omega)$ of half-widths at half-maximum $\Gamma_{i}(Q)$ as defined in Section 2.3. The experimentally determined $A_{i}(Q)$ are related to the areas $B_{i}(Q)$ in Eqs.(8) and (9) by expressions derived in Appendix $\mathrm{C}$ and given in Table 3 . $\mathcal{R}(Q, \omega)$ refers to the resolution function and corresponds to the Vanadium measurements directly included in the analysis and $\mathcal{B}(Q)$ is a flat background, that can include the instrument contribution, or fast vibrational motions outside the window. 
Table 5: Relaxation rates of dynamical processes.

\begin{tabular}{ll}
\hline \hline Subunit & Relaxation rates ${ }^{(a)}$ \\
\hline \hline C-H groups & $\begin{array}{l}\text { jump dynamics between } 2 \text { non-equivalent sites distant from d } \\
\Gamma_{\text {jump }}\end{array}$ \\
\hline Tail & 2d-diffusion of tail H-atoms inside $F(m)$ - distribution of circles of radius $R_{m}$ \\
& ${ }^{(b)} \Gamma_{\text {tail }}(Q)=D_{\text {tail }}\left[\sum_{m=1}^{M} F(m)\left(1-A_{\text {tail }}(Q)\right)\left[\frac{\left(x_{0}^{1}\right)^{2}}{R_{m}^{2}}+Q^{2}\right]\right] /\left[\sum_{m=1}^{M} F(m)\left(1-A_{\text {tail }}(Q)\right)\right]$ \\
\hline Head & rotational diffusion of the headgroup about its axis plus flip-flop motion \\
& $\Gamma_{\text {head }}=D_{\text {head }}+\frac{1}{\tau_{\mathrm{ff}}}$ \\
& rotational diffusion of the lipid molecule about the membrane normal axis \\
& $\Gamma_{\text {rot }}=D_{\text {rot }}$ \\
& in-out of the plane $1 d$-diffusion of the lipid molecule in a harmonic potential \\
& $\left(\right.$ c) $\Gamma_{\text {in-out }}(Q)=\left[(Q \text { cos } \gamma)^{2}\left(\frac{k_{\mathrm{B}} T}{k}\right) \frac{1}{\tau}\right] /\left[1-\right.$ exp $\left.\left\{-(Q \cos \gamma)^{2}\left(\frac{k_{\mathrm{B}} T}{k}\right)\right\}\right]$ \\
& in-plane $2 d$-diffusion of the lipid molecule inside a circle of radius $R_{\|}$ \\
& $\Gamma_{2 \mathrm{~d}}=\left[\frac{\left(x_{0}^{1}\right)^{2}}{R_{\|}^{2}}+Q^{2}\right] D_{\|}$
\end{tabular}

${ }^{(a)}$ Following Ref.[16], the relaxation rate for each dynamical process is given by, $\frac{1}{\Gamma_{\alpha}(Q)}=$ $\int_{0}^{\infty} d t\left[\frac{I_{\alpha}(Q, t)-A_{\alpha}(Q)}{1-A_{\alpha}(Q)}\right]$ for $\alpha=$ jump, head, tail, rot, in - out, 2d. For the simplicity of calculations, the $\Gamma_{\alpha}$ reported in this table are derived at short time limit as, $\Gamma_{\alpha}(Q)=-\lim _{t \rightarrow 0} \frac{d}{d t}\left[\frac{I_{\alpha}(Q, t)-A_{\alpha}(Q)}{1-A_{\alpha}(Q)}\right]$. (b) where $x_{0}^{1}=1.84118$. Note that $\Gamma_{\text {tail }}(Q)=\left(x_{0}^{1}\right)^{2} \frac{D_{\text {tail }}}{\left\langle R_{m}^{2}\right\rangle}$ in the $Q \rightarrow 0$ limit and $\Gamma_{\text {tail }}(Q)=Q^{2} D_{\text {tail }}$ in the $Q \rightarrow \infty$ limit, where $\left\langle R_{m}^{2}\right\rangle=(M+1) R_{1}^{2} / 2$ for $F(m)=1 / M$ and $R_{m}=R_{1} \sqrt{m}$.

(c) Note that $\Gamma_{\text {in-out }}(Q)=1 / \tau$ in the $Q \rightarrow 0$ limit and $\Gamma_{\text {in-out }}(Q) \approx(Q \cos \gamma)^{2}\left(\frac{k_{\mathrm{B}} T}{k}\right) \frac{1}{\tau}$ in the $Q \rightarrow \infty$ limit. 
The areas retrieved from the QENS analyses were fitted with the A's in Table 3 using the package $l m f i t$ from Python [20] with Levenberg-Marquardt and Nelder-Mead algorithms. Fitting procedure relies on the simultaneous fitting of the four areas with corresponding functions given in Tables 3 and 4 through a set of shared parameters. In this way, both statistics are improved with more data points included and constraints on parameters are controlled.

\subsection{Results}

Main results within the framework of this analysis can be summarized as follows:

- Data from QENS experiments are analyzed as illustrated in Fig. 3 where the Eq.(11) is used to fit the data points and extract both the experimental Lorentzian amplitudes (areas) $A_{i}(Q)$ 's and HWHM $\Gamma_{i}(Q)$ 's. Figure 3 shows that the 3-dynamical process model describes very well (with residuals $\sim 0$ ) experimental data into an elastic peak $\left(\delta(\omega)\right.$, a Lorentzian with $\Gamma_{0}=0$, and amplitude $\left.A_{0}(Q)\right)$ plus three well separated Lorentzians (with, $\Gamma_{1} \sim 0.1 \mathrm{meV}, \Gamma_{2} \sim 1 \mathrm{meV}$ and $\Gamma_{3} \sim 10 \mathrm{meV}$, for slow, intermediate and fast motions, respectively; there is an order of magnitude between the $\Gamma_{i}$ 's). Such an analysis is performed for all values of the pair $(Q, T)$ considered in the experiment.

In the rest of this illustrative example, we will only deal with the $A_{i}(Q)$ 's for a single sample in a given geometry whereas the use of this approach on several samples of lipid systems in different geometries with the analysis including $S(Q, \omega), A_{i}(Q)$ and $\Gamma_{i}(Q)$ will be presented and discussed in more details elsewhere [21, 22]. Therefore, the number of parameters to determine is reduced to 11 for the Matryoshka model plus the 2 experimental parameters $\left(m\right.$ and $\left.\varepsilon_{0}\right)$. All the parameters are listed in the Table 6 where some are fixed (as extracted from the literature) and others are obtained from the best fit of the model to experimental data.

- Experimental Lorentzian amplitudes (areas) $A_{i}(Q)$ 's extracted as described above in Fig. 3 are represented by data points in Fig. 4. We observe that the $A_{i}(Q)$ exhibit as a function of $Q$ a behavior exactly as predicted by the model (see the end of the Section 2.3): $A_{0}(Q) \sim 1$ while $A_{i>0}(Q) \sim 0$ at low $Q \rightarrow 0$ as expected for spatially restricted motions, $A_{0}(Q)$ and $A_{1}(Q)$ decreases to very low value (closed to zero) in the large $Q$ limit like for continuous motions while $A_{2}(Q)$ and $A_{3}(Q)$ increase with $Q$ to a non-zero value reflecting the spatially discrete motions of the backbone.

To go further in the analysis of these data, expressions of the amplitudes as a function of $\mathrm{Q}$ are needed; this is what we have done in deriving the expressions given in Tables 3 and 4. Lines through the data in Fig. 4 represent best fits (with $\chi^{2} \sim 1.5$ ) of $A_{i}(Q)$ 's using expressions in Tables 3 and 4 with the physical parameters of the local motions thus determined and reported in Table 6. This figure clearly demonstrates the relationships between local motions and the amplitudes of the phenomenological 3-dynamical process model. 


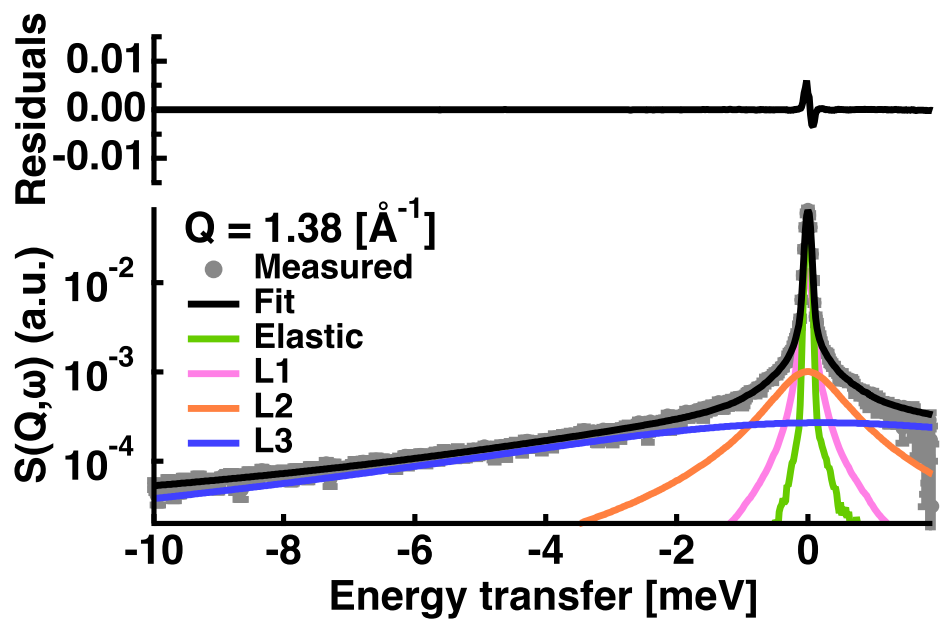

Figure 3: Example of $S\left(Q=1.38 \AA^{-1}, \omega\right)$ data fitting for DMPC multilamellar bilayers sample measured on IN6 at $135^{\circ}$ geometry (in-plane motions) and $\mathrm{T}=280 \mathrm{~K}$. Solid black line through data points (grey circles) represents the best fit to the data using Eq.(11) with the resolution function given by the Vanadium measurements at $\mathrm{Q}=1.38 \AA^{-1}$. The fit results from the sum of the elastic peak (green line) and of the three Lorentzian functions for slow (magenta line), intermediate (orange line) and fast (blue line) motions.

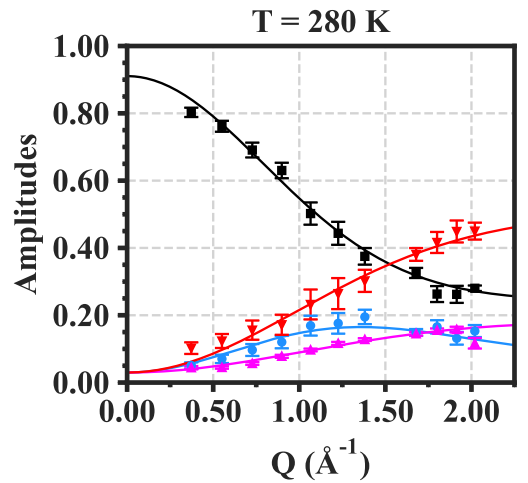

I $\quad \mathbf{A}_{0}$ (elastic)

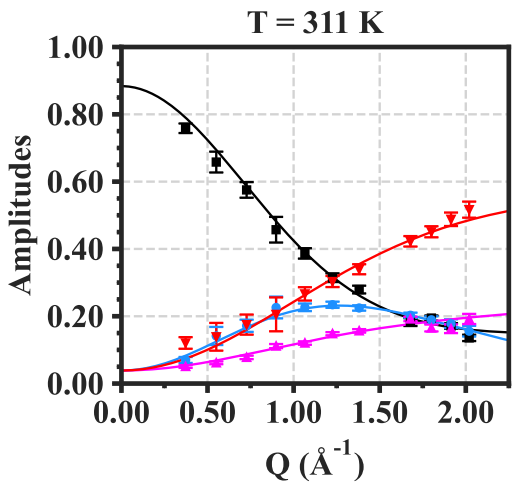

I $\quad \mathbf{A}_{1}$ (slow)

I $\quad \mathbf{A}_{2}$ (intermediate)

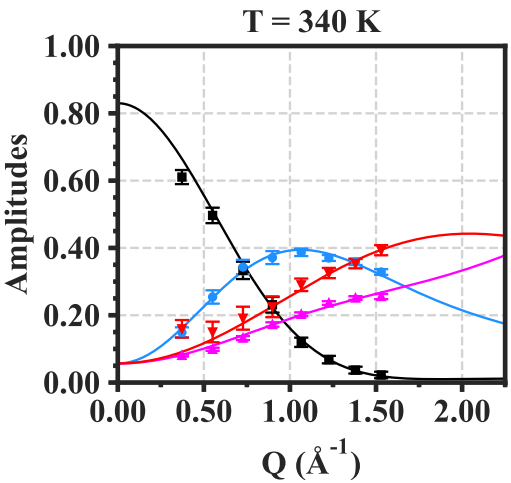

I $\quad \mathbf{A}_{3}$ (fast)

Figure 4: Data fitting for DMPC multilamellar bilayers measured at $135^{\circ}$ (in-plane motions). Data points correspond to experimental amplitudes (areas) of the 3-dynamical process as a function of $Q$ for different temperatures, obtained from best fits of QENS data with Eq.(11) as illustrated in Figure 3. Solid lines through the data represent best fits to the data using the expressions of amplitudes given in Tables 3 and 4. Parameters extracted from these fits are reported in Table 6. 
Table 6: Fixed and Fitted parameters for DMPC MLBs. The sample was measured on IN6 instrument at $135^{\circ}$ (in-plane motions or $\|$ geometry).

\begin{tabular}{|c|c|c|c|c|c|c|c|}
\hline Id & Parameter & Definition & $T=280 K$ & $\mathrm{~T}=311 \mathrm{~K}$ & $\mathrm{~T}=340 \mathrm{~K}$ & Units & Reference \\
\hline 1 & $\left\langle u^{2}\right\rangle$ & $\begin{array}{l}\text { H's mean-squared } \\
\text { displacement }\end{array}$ & $0.83 \pm 0.05$ & $1.30 \pm 0.06$ & $5.06 \pm 0.43$ & $\AA^{2}$ & Fitted \\
\hline \multirow{2}{*}{2} & $d$ & two-site distance & $2.2 \pm 0.1$ & $2.2 \pm 0.1$ & $2.2 \pm 0.1$ & $\AA$ & {$[23,24]^{(a)}$} \\
\hline & $\phi$ & probability of jump events & $0.06 \pm 0.02$ & $0.07 \pm 0.01$ & $0.26 \pm 0.02$ & - & Fitted \\
\hline \multirow{3}{*}{3} & $1-z$ & fraction of H's in the tail & 0.75 & 0.75 & 0.75 & - & {$[25]$} \\
\hline & $M$ & length of the effective tail & 14 & 14 & 14 & - & {$[25]$} \\
\hline & $R_{1}$ & radius of the first circle & $0.43 \pm 0.04$ & $0.43 \pm 0.02$ & $0.01 \pm 0.40$ & $\AA$ & Fitted \\
\hline \multirow{2}{*}{4} & $\alpha$ & head tilt angle & $32.3 \pm 0.6$ & $32.3 \pm 0.6$ & $32.3 \pm 0.6$ & $\circ$ & {$[26,27]$} \\
\hline & $b_{\mathrm{H}}$ & headgroup H-radius & $1.04 \pm 0.04$ & $1.16 \pm 0.04$ & $0.66 \pm 0.08$ & $\AA$ & Fitted \\
\hline 5 & $R_{\mathrm{H}}$ & H-radius of the lipid & $0.01 \pm 0.42$ & $0.46 \pm 0.01$ & $0.62 \pm 0.01$ & $\AA$ & Fitted \\
\hline 6 & $k$ & force constant & No & No & No & $\mathrm{N} / \mathrm{m}$ & Fitted \\
\hline 7 & $R_{\|}$ & lipid cage radius & $1.13 \pm 0.08$ & $1.38 \pm 0.04$ & $2.06 \pm 0.06$ & $\AA$ & Fitted \\
\hline \multirow{2}{*}{8} & $m$ & $\%$ of dynamic H's & $67 \pm 1$ & $73 \pm 2$ & $82 \pm 1$ & - & Fitted \\
\hline & $\varepsilon_{0}$ & error term & $0.24 \pm 0.01$ & $0.15 \pm 0.01$ & $0.01 \pm 0.01$ & - & Fitted \\
\hline
\end{tabular}

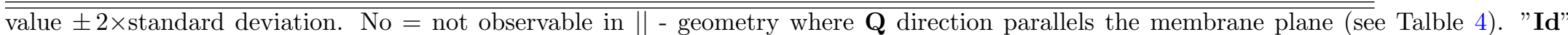
stands for motions with: $1=$ vibrational motions, $2=$ jump dynamics of the backbone, $3=$ tails: diffusion in circles, $4=$ head: rotational diffusion+filp-flop, $5=$ rotational diffusion of the lipid molecule, $6=$ In-out of the plane diffusion, $7=$ in-plane $2 \mathrm{~d}$-diffusion, and $8=$ experimental parameters.

(a) Mean distance between the two sites for the jump diffusion of C-H groups. From Ref.[23]: go to Home $\rightarrow$ Geometry $\rightarrow$ Experimental $\rightarrow$ Internal coordinates or Bondangles, and look for the HCH to obtain the average angle $\widehat{H C H}$ (https://cccbdb.nist.gov/expangle2x.asp? descript=aHCH\&all=0), the experimental length $d_{\mathrm{CH}}$ of $\mathrm{C}-\mathrm{H}($ https $: / /$ cccbdb.nist.gov/expbondlengths $2 \mathrm{x}$.asp?descript=rCH\&all=0) and calculate the distance $d$ as, $d=2 d_{\mathrm{CH}} \sin (\widehat{H C H} / 2)$. See also, Ref.[24], p.88. 
- From now on, we will be able to take an interest in local motions. Figure 5 shows the EISF's or amplitudes (except the Debye-Waller factor) of all local motions contributing to the 3-dynamical process. These EISF's are generated by using parameters determined from Fig. 4 into expressions in Table 4 . Changes with temperature of the variations in amplitudes as a function of $Q$ reflect changes of parameters with temperature (see Table 6). Here, we did not nor have developed models to predict how these parameters might change with temperature. We can already notice that in general all parameters exhibit an increase with temperature (see Table 6). Indeed, the lipid system gains thermal energy as temperature increases leading to a subsequent enhancement of the dynamics, especially above the main phase transition around $297 \mathrm{~K}$. Interestingly, the Matryoshka model already proves to be sensitive enough to allow capturing differences between the gel and liquid phase in such lipid systems.

In terms of motion amplitudes, observable mouvements have amplitudes $A_{i}(Q)$ deviating from the horizontal line 1 as a function of $Q$. The deviation increases when $A_{i}(Q)$ decreases (i.e., the extent of motions increases) and vice versa, i.e., the area above the amplitude $(F), F_{i}=\left(1 / Q_{\max }\right) \int_{0}^{Q_{\max }}\left[1-A_{i}(Q)\right] d Q$, increases with the extension or amplitude of motions. More specifically,

$\triangleright$ Fraction of dynamic H's: Values of $m$ in Table 6 (given in \%) are similar to that found in the literature [28], with $m$ increasing with temperature.

$\triangleright$ Individual motions: The Debye-Waller factor is a gaussian function of $Q$ (not shown in Fig. 5) with the mean-square displacements (very similar to that found in literature [28]) increasing with temperature (see Table 6). As a result, $F_{\mathrm{DW}}(Q)$ increases with temperature.

$\triangleright$ Internal motions

$\rightarrow$ Backbone motions: Backbone motions relate to movements of $\mathrm{C}-\mathrm{H}$ groups described by the jump diffusion between two non-equivalent sites distant of $d$. As $d$ is fixed (see Table 6), the change in the amplitude of backbone motions with temperature reflects the change in the probability $\phi$ of jump events that increases with temperature (see Table 6 ); note that $\phi(T=340 K) \approx 0.26$ whereas $\phi_{\max }=0.5$. Likewise, Fig. 5 shows that the amplitude of backbone motions increases as $F_{\text {jump }}(Q)$ increases with temperature.

$\rightarrow$ Tail motions: Figure 5 shows that the diffusional motions of tails are clearly observables with almost same amplitudes for temperatures $T=280 \mathrm{~K}$ and $T=311 K$ as reflected by values of $R_{1}$ in Table 6 . And at $T=340 \mathrm{~K}$, the $A_{i}(Q)$ of tail motions exhibit an increase and become similar to that of backbone motions. This behavior which does not go in the direction of the increase of parameters with temperature finds its origin in the imprecise value of $R_{1}$ with large error bars (Table 6 ). It indicates possible variations of other parameters of the motion with temperature and / or relations between $R_{1}$ and others parameters. Further analysis would be needed. 
$\rightarrow$ Head motions: Figure 5 shows that the amplitude of the head motions slightly increases with temperature at low temperature then decreases at higher temperature as reflected by changes in the head size $b_{\mathrm{H}}$ (see Table 6). Such a change of $b_{\mathrm{H}}$ at higher temperatures is interesting to note, especially since it could be indicative of an interference in the analysis between internal rotational motions of the heads around their axes and the molecule rotational movements of lipid molecules (see Rotational motions and Figure 5); both involving the H-atoms in the head group. More analysis would be needed. For example, analyzing amplitudes in $\perp$ geometry, where $\mathbf{Q}$ is perpendicular to the membrane and only the movements of the heads are observable but not those of molecule rotation (see Table 4), could give informative indications on how to disentangle the motions.

$\triangleright$ Molecule motions

$\rightarrow$ Rotational motions: It appears that the amplitude of the rotational motions of lipid molecules about lipid axis are weak $\left(A_{\text {rot }}(Q) \sim 1\right)$ at low temperature and increases $\left(A_{\text {rot }}(Q)<1\right)$ when the temperature increases thus resulting in an increase of the H-radius $R_{\mathrm{H}}$ of lipid molecules with temperature (see Table 6).

$\rightarrow$ In-out the plane motions: The in-out of the plane motions of lipid molecules are not observable for the $\|$ geometry where the neutron scattering vector $\mathbf{Q}$ is parallel to the membrane (see Table 4). In this case, the amplitude remains the horizontal line, $F_{\text {in-out }}(Q)=0$, for all pairs of $(Q, T)$ as shown in Fig. 5.

$\rightarrow$ In-plane $2 d$ diffusion motions: The in-plane diffusion of lipid molecules occurs within a cage of radius $R_{\|}$formed by the neighboring lipid molecules. Figure 5 shows that the deviation $F_{2 \mathrm{~d}}(Q)$ increases as the temperature gets higher, thus indicating that $R_{\|}$increases with the temperature (see Table 6 ).

Finally, let us underline that some parameters of the model in the Table 6 are not comparable with structure parameters which could have been obtained from diffraction experiments, for example. Indeed, unlike the structural parameters, the model parameters relate to the local movements of the $\mathrm{H}$ atoms around their equilibrium positions. By definition, these dynamical parameters are different and will generally be smaller than their structural counterparts. For example, the values of the H-radius, $b_{\mathrm{H}}$, of the head group are slightly smaller than the experimental data $(\sim 2 \AA)$ of the head size [26, 29]. Likewise, the values of the smallest radius $R_{1}$ for the tail motions are smaller than the experimental values $(\sim 2.2 \AA)$ of chain spacings at the start of the tails $[26,29]$. As for the H-radius, $R_{\mathrm{H}}$, it can be compared to the span of the lipid molecule given by $D_{\mathrm{H} 1} \sin (\alpha)$, where $D_{\mathrm{H} 1}$ (similar but different from $d_{\mathrm{H}}$ in Fig. 2) is the size of the head group and $\alpha$ the tilt angle of the head. We find that $R_{\mathrm{H}}$ is smaller than the lipid span for $\alpha$ given in Table 6 and $D_{\mathrm{H} 1} \sim 5 \AA$ obtained from diffraction $[26,29]$. 

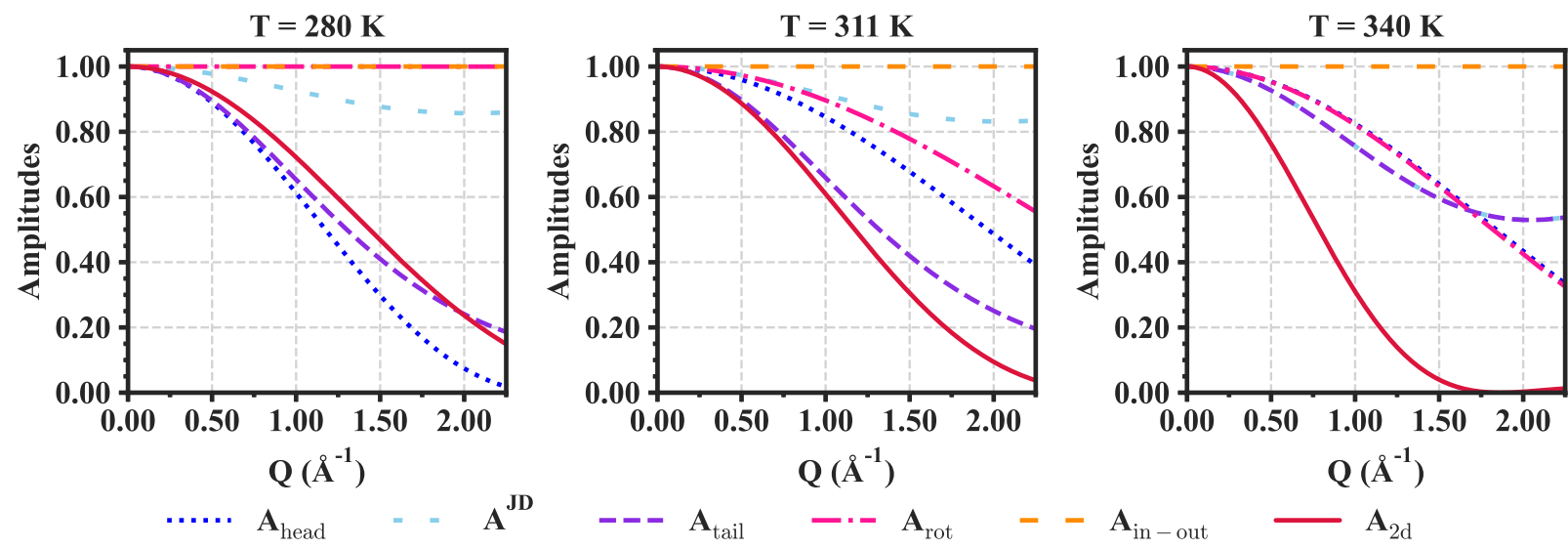

Figure 5: EISF or amplitudes of local motions (contributing to the 3-dynamical process) resolved in DMPC multilamellar bilayers at $135^{\circ}$ (in-plane motions) as a function of $Q$ for different temperatures. Lines correspond to expressions in Table 4 using parameters from Table 6.

\section{Concluding summary}

Our main motivation in developing this work has been to construct a framework for studying and describing local dynamics of lipid molecules in membrane layers. The main results of this work can be summarized as follows:

- We have developed the dynamical Matryoshka model which describes the local dynamics of lipid molecules in a membrane layer as a nested hierarchical convolution of three motional processes (see, Table 1):

(i) individual motions described by vibrational movements of H-atoms;

(ii) internal motions including the motions of the lipid backbone described by a jump dynamics between two non-equivalent sites, the head motions described by a rotational diffusion about the head axis plus a flip-flop or jump dynamics of the head tilted angle about the lipid axis, and the motions of the tail described by two-dimensional diffusions of $\mathrm{H}$-atoms within circles of increasing radii along the tails;

(iii) molecule motions of the lipid molecule as a whole including the rotational diffusion about the lipid axis, in-out of the plane motion and the in-plane local diffusion of the lipid molecule within a cage.

The model includes seven motions in total (see, Table 2) with the analytical expressions of all associated ISFs provided in Table A.7.

- For the purpose of analyzing QENS experimental data, we have derived an analytical expression for the aggregated ISF of the Matryoshka model which involves an elastic term plus three inelastic terms of well-separated time scales. In doing so, we obtain the relationships between the amplitudes and rates of the aggregated ISF and those of the local movements of the initial model (see, Table 3). 
- As a check of the model, we have shown that the theoretical aggregated ISF fit very well the QENS experimental data on a DMPC sample and allow extracting the dynamical parameters of the model.

Although we have shown that the development outlined above already describes very well QENS experiments, it can be complemented with analyzes including both the amplitudes, $A_{i}(Q)$, and rates, $\Gamma_{i}(Q)$, for various samples of lipid systems in different geometries. Such work is carried out in $[21,22]$. The Matryoshka model provides a framework within which several improvements can be carried out if necessary. For example, for local motions, we have described the motions of the backbone by a jump dynamics between two sites. It is quite possible to replace it with other movements (e.g., jump dynamics between three sites) that the associated EISF (which contributes in the amplitudes of intermediate and fast motions) does not cancel at large $Q$, as experimental evidence indicates that the movements of the backbone should be spatially discrete. The Matryoshka model outlined above can be further extended to include in the same setting long range jumps for in-plane lipid diffusion and collective undulation modes of lipids. In terms of the time scales discussed here, these motions belong to the class of slow or very slow motions ( $\Gamma \sim 1 \mu \mathrm{eV})$. Such a work is in progress. Finally, to be complete in the analysis of QENS data, it might turn out necessary to develop models predicting how the parameters of local motions would change with temperature, for example.

\section{Acknowledgements}

The authors thank the Institut Laue-Langevin for the allocation of the beam time to perform the experiments. We also thank Francesca Natali for her help on the experimental aspects of QENS. AC is supported by the JP Aguilar scholarship from Fondation CFM for her $\mathrm{PhD}$ thesis.

\section{Data Availability}

The datasets generated and analyzed during the current study are available from the corresponding author on reasonable request.

\section{Author Contributions}

Dominique J. Bicout: Conceptualization, Methodology, Writing - Original Draft, Review \& Editing, Supervision. Aline Cisse: Software, Validation, Formal Analysis, Writing Review \& Editing. Tatsuhito Matsuo: Software, Validation, Formal Analysis, Writing Review \& Editing. Judith Peters: Investigation, Writing - Review \& Editing, Supervision, Funding acquisition.

\section{Competing Interests:}

The authors declare that they have no financial or non-financial competing interests. 


\section{Appendix A. Derivations of expressions of ISF}

The key quantity in neutron scattering is the incoherent structure function (ISF), defined as,

$$
I(Q, t)=\left\langle e^{i \mathbf{Q} \cdot \mathbf{r}(\mathbf{t})} e^{-i \mathbf{Q} \cdot \mathbf{r}(\mathbf{0})}\right\rangle
$$

where $\mathbf{Q}$ is the neutron scattering wave vector, $\mathbf{r}(\mathbf{t})$ and $\mathbf{r}(\mathbf{0})$ the hydrogen atom positions at $t=0$ and time $t$, respectively, and the sign $\langle\cdots\rangle$ represents an ensemble average over all positions $\mathbf{r}(\mathbf{t})$ and $\mathbf{r}(\mathbf{0})$ in the potential of mean force, $V(\mathbf{r})$. The mathematical expressions of all the ISFs considered in this work are gathered in Table A.7. For the ISFs whose expressions had already been derived elsewhere, we have indicated the associated references, and for the others we derive the expressions in this Appendix. This will be the case for head rotational movements with flipflop and the molecular in-out-of the plane diffusion of the lipid molecule in a harmonic potential. 
Table A.7: Incoherent Structure Functions (ISF) of dynamical processes.

\begin{tabular}{|c|c|c|}
\hline Subunit & ISF & Mathematical expression \\
\hline H-atom & ${ }^{(a)} I_{\mathrm{DW}}$ & $\begin{array}{l}\text { vibrational motions: Debye-Waller factor } \\
I_{\mathrm{DW}}(Q, t)=\exp \left\{-\frac{Q^{2}\left\langle u^{2}\right\rangle}{3}\right\}\end{array}$ \\
\hline C-H groups & ${ }^{(b)} I_{\text {jump }}$ & $\begin{array}{l}\text { jump dynamics between } 2 \text { non-equivalent sites distant from } d \\
I_{\text {jump }}(Q, t)=A_{\text {jump }}(Q)+\left[1-A_{\text {jump }}(Q)\right] \exp \left\{-\Gamma_{\text {jump }}|t|\right\}, A_{\text {jump }}(Q)=1-2 \phi(1-\phi)\left[1-j_{0}(Q d)\right]\end{array}$ \\
\hline Tail & (c) $I_{\text {tail }}$ & $\begin{array}{l}\text { 2d-diffusion of tail H-atoms inside } F(m) \text { - distribution of circles of radius } R_{m} \\
\begin{array}{l}I_{\text {tail }}(Q, t)=\sum_{m=1}^{M} F(m)\left\{A_{0}^{0}\left(y_{m}\right)+\sum_{n=1}^{+\infty} A_{n}^{0}\left(y_{m}\right) \exp \left\{-\left(x_{n}^{0}\right)^{2}\left(D_{\text {tail }} / R_{m}^{2}\right)|t|\right\}\right. \\
\left.\quad+2 \sum_{l=1}^{+\infty} \sum_{n=0}^{+\infty} A_{n}^{l}\left(y_{m}\right) \exp \left\{-\left(x_{n}^{l}\right)^{2}\left(D_{\text {tail }} / R_{m}^{2}\right)|t|\right\}\right\} \\
A_{0}^{0}\left(y_{m}\right)=\left[\frac{2 J_{1}\left(y_{m}\right)}{y_{m}}\right]^{2} ; A_{n}^{l}\left(y_{m}\right)=\frac{4\left(x_{n}^{l}\right)^{2}}{\left(x_{n}^{l}\right)^{2}-l^{2}} \cdot\left[\frac{y_{m} J_{l+1}\left(y_{m}\right)-l J_{l}\left(y_{m}\right)}{y_{m}^{2}-\left(x_{n}^{l}\right)^{2}}\right]^{2} ; y_{m}=Q R_{m} \sin \gamma\end{array}\end{array}$ \\
\hline
\end{tabular}

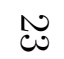

${ }^{(d)} I_{\text {head }}$ rotational diffusion of the headgroup about its axis plus flip-flop motion

Head

$I_{\text {head }}(Q, t)=\sum_{n=-\infty}^{+\infty} A_{n}(Q) \exp \left\{-\left[n^{2} D_{\text {head }}+\frac{1-(-1)^{n}}{2 \tau_{\mathrm{ff}}}\right]|t|\right\}$

$A_{n}(Q)=\left[\sum_{l=-\infty}^{+\infty} J_{n-2 l}\left(Q b_{\mathrm{H}} \cos \gamma \sin \alpha\right) J_{l}\left(Q b_{\mathrm{H}} \sin \gamma \cos ^{2} \frac{\alpha}{2}\right) J_{l}\left(Q b_{\mathrm{H}} \sin \gamma \sin ^{2} \frac{\alpha}{2}\right)\right]^{2}$

(e) $I_{\text {rot }} \quad$ rotational diffusion of the lipid molecule about the membrane normal axis $I_{\text {rot }}\left(Q R_{\mathrm{H}} \sin \gamma, t\right)=J_{0}^{2}\left(Q R_{\mathrm{H}} \sin \gamma\right)+2 \sum_{n=1}^{+\infty} J_{n}^{2}\left(Q R_{\mathrm{H}} \sin \gamma\right) \exp \left\{-D_{\mathrm{rot}} n^{2}|t|\right\}$

Molecule $\quad{ }^{(f)} I_{\text {in-out }}$ in-out of the plane 1d-diffusion of the lipid molecule in a harmonic potential $I_{\text {in-out }}(Q \cos \gamma, t)=\exp \left\{-(Q \cos \gamma)^{2}\left(\frac{k_{\mathrm{B}} T}{k}\right)\left[1-\mathrm{e}^{-|t| / \tau}\right]\right\}$

${ }^{(g)} I_{2 \mathrm{~d}} \quad$ in-plane 2d-diffusion of the lipid molecule inside a circle of radius $R_{\|}$

$$
\begin{aligned}
& I_{2 \mathrm{~d}}\left(Q R_{\|} \sin \gamma, t\right)=A_{0}^{0}(y)+\sum_{n=1}^{+\infty} A_{n}^{0}(y) \exp \left\{-\left(x_{n}^{0}\right)^{2}\left(D_{\|} / R_{\|}^{2}\right)|t|\right\}+2 \sum_{l=1}^{+\infty} \sum_{n=0}^{+\infty} A_{n}^{l}(y) \exp \left\{-\left(x_{n}^{l}\right)^{2}\left(D_{\|} / R_{\|}^{2}\right)|t|\right\} \\
& A_{0}^{0}(y)=\left[\frac{2 J_{1}(y)}{y}\right]^{2} ; A_{n}^{l}(y)=\frac{4\left(x_{n}^{l}\right)^{2}}{\left(x_{n}^{l}\right)^{2}-l^{2}} \cdot\left[\frac{y J_{l+1}(y)-l J_{l}(y)}{y^{2}-\left(x_{n}^{l}\right)^{2}}\right]^{2} ; y=Q R_{\|} \sin \gamma
\end{aligned}
$$

(1)


(a) corresponds to the long time limit as the relaxation of vibrational motions is very fast and out of the time scales considered here. $\left\langle u^{2}\right\rangle$ is the mean square amplitude of these very fast motions.

(b) $j_{0}(\cdots)$ is the spherical Bessel function of firts kind and order $0, \phi=\tau_{1} /\left(\tau_{1}+\tau_{2}\right)=1 /\left[1+\exp \left(-\Delta G / k_{\mathrm{B}} T\right)\right]$ and $\Gamma_{j u m p}=\tau_{1}^{-1}+\tau_{2}^{-1}$ where $\tau_{1}$ and $\tau_{2}$ are the mean residence times in each site and $\Delta G$ the standard energy variation of the transition between the two sites [14].

(c) See ${ }^{(g)}$ for the derivation. $D_{\text {tail }}$ is the diffusion constant. The simplest distribution law is, $F(m)=1 / M$, where $M$ is the apparent chain length of the lipid tail, and the circle-radius $R_{m}=R_{1} \sqrt{m}$. Carpentier et al. [15] rather used a two-parameters model for the circle-radius, $R_{m}=\left(\frac{m-2}{M-2}\right)\left(R_{M}-R_{2}\right)+R_{2}$.

(d) $b_{\mathrm{H}}$ is the H-radius of the head group (i.e., the distance between the head axis and the center of inertia of all H-atoms of the head group with respect to the head axis $\vec{l}$ ), $\alpha$ the tilt angle between the membrane normal axis and head axis (see Fig.2), $D_{\text {head }}$ the head rotational diffusion constant and $\tau_{\mathrm{ff}}$ the mean resting time between two successive flip-flops. $J_{n}(\cdots)$ is the cylindrical Bessel function of first kind and order $n$. See the derivation in Sec. Appendix A.1.

(e) See Eq.(13) in Ref.[30]. The size of the cross section of the lipid molecule is given by, $R_{\mathrm{H}}$ (i.e., the distance between the lipid axis and the center of inertia of all dynamic H-atoms with respect to the lipid axis), $\gamma$ the angle between $\mathbf{Q}$ and the membrane normal axis and $D_{\text {rot }}$ the rotational diffusion constant.

(f) $k$ is the force constant, $\tau$ the relaxation time, $k_{\mathrm{B}} T$ the thermal energy with $k_{\mathrm{B}}$ the Boltzmann constant and $T$ temperature. See the derivation in Appendix A.2.

(g) See the derivation in Ref.[31]. $J_{l}(\cdots)$ is the cylindrical Bessel function of first kind and order $l$ and $x_{n}^{l}$ is the $(n+1)$ th root of, $\stackrel{d}{\vDash}\left[J_{l}(x)\right]=0$; these roots are tabulated in [32], e.g., $x_{n}^{0}=3.83170,7.01558,10.17346$ for $n=1,2,3$ and $x_{n}^{1}=1.84118,5.33144,8.53632$ for $n=0,1,2$, where $x_{0}^{1}$ is the smallest non-zero root. $D_{\|}$is the diffusion constant. 


\section{Appendix A.1. Rotational diffusions and flip-flop of headgroup}

We will consider the head group as an ellipsoid of circular cross-section of diameter $2 b_{\mathrm{H}}$ and directing vector $\vec{l}$ (see Fig.2), where $b_{\mathrm{H}}$ is the distance between the head axis and the center of inertia of all H-atoms of the head group with respect to the head axis $\vec{l}$ and $\vec{l}$ is given in the orthonormal frame $\widehat{x y z}$ of basis $(\vec{i}, \vec{j}, \vec{k})$ by,

$$
\vec{l}=\left(\begin{array}{c}
\sin \alpha \cos \eta \\
\sin \alpha \sin \eta \\
\cos \alpha
\end{array}\right)
$$

The angles are defined in Fig.A.6.

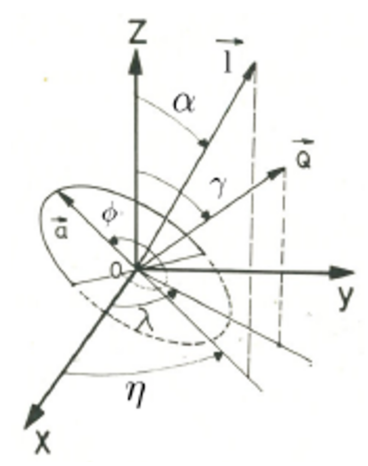

Figure A.6: Definition of angles for the rotations about $\vec{n}$ (parallell to $z$ ) and $\vec{l}$ directions and the flip-flop motion of $\vec{l}$ about $\vec{n}$. Adaptation from [30].

That thus makes it possible to define a local orthonormal coordinate system $\left(\vec{e}_{x}, \vec{e}_{y}, \vec{e}_{z}\right)$ associated with the head group as follows,

$$
\left\{\begin{array}{l}
\vec{e}_{x}=(\cos \alpha \cos \eta) \vec{i}+(\cos \alpha \sin \eta) \vec{j}-(\sin \alpha) \vec{k} \\
\vec{e}_{y}=-(\sin \eta) \vec{i}+(\cos \eta) \vec{j} \\
\vec{e}_{z}=\vec{l}=(\sin \alpha \cos \eta) \vec{i}+(\sin \alpha \sin \eta) \vec{j}+(\cos \alpha) \vec{k}
\end{array}\right.
$$

from which the matrix of passage from $\left(\vec{e}_{x}, \vec{e}_{y}, \vec{e}_{z}\right)$ to $(\vec{i}, \vec{j}, \vec{k})$ can be determined as,

$$
P=\left[\vec{e}_{x}, \vec{e}_{y}, \vec{e}_{z}\right]=\left(\begin{array}{ccc}
\cos \alpha \cos \eta & -\sin \eta & \sin \alpha \cos \eta \\
\cos \alpha \sin \eta & \cos \eta & \sin \alpha \sin \eta \\
-\sin \alpha & 0 & \cos \alpha
\end{array}\right)
$$

Note that $P=R_{z}(\eta) R_{y}(\alpha)$ results from the operation of two rotations where $R_{y}(\alpha)$ describes the rotation of an angle $\alpha$ about $y$-axis and $R_{z}(\eta)$ the rotation of an angle $\eta$ about $z$-axis. The head group position vector $\vec{b}$ in the local head group frame $\left(\vec{e}_{x}, \vec{e}_{y}, \vec{e}_{z}\right)$ is given by,

$$
\vec{b}=b_{\mathrm{H}}\left(\begin{array}{c}
\cos \phi \\
\sin \phi \\
0
\end{array}\right)=b_{\mathrm{H}}\left[(\cos \phi) \vec{e}_{x}+(\sin \phi) \vec{e}_{y}\right]
$$


where $\phi$ is the angle between $\vec{b}$ and $\vec{e}_{x}$. Thus, the head group position vector $\mathbf{r}$ in the frame $(\vec{i}, \vec{j}, \vec{k})$ is obtained using the passage matrix as,

$$
\begin{aligned}
\mathbf{r} & =P \vec{b}=b_{\mathrm{H}}\left(\begin{array}{ccc}
\cos \alpha \cos \eta & -\sin \eta & \sin \alpha \cos \eta \\
\cos \alpha \sin \eta & \cos \eta & \sin \alpha \sin \eta \\
-\sin \alpha & 0 & \cos \alpha
\end{array}\right)\left(\begin{array}{c}
\cos \phi \\
\sin \phi \\
0
\end{array}\right) \\
& =b_{\mathrm{H}}\left(\begin{array}{c}
\cos \alpha \cos \eta \cos \phi-\sin \eta \sin \phi \\
\cos \alpha \sin \eta \cos \phi+\cos \eta \sin \phi \\
-\sin \alpha \cos \phi
\end{array}\right)
\end{aligned}
$$

Note that, $\mathbf{r} \cdot \vec{l}=0$ as expected since $\mathbf{r} \perp \vec{l}$. by,

Let the $z$-axis be the normal to the membrane, the scattering wave vector $\mathbf{Q}$ is given

$$
\mathbf{Q}=Q\left(\begin{array}{c}
\sin \gamma \cos \lambda \\
\sin \gamma \sin \lambda \\
\cos \gamma
\end{array}\right)
$$

where $\gamma$ is the angle between $\vec{k}$ and $\mathbf{Q}$ and $\lambda$ the angle between the projection of $\mathbf{Q}$ onto the $x-y$ plan and $\vec{i}$. Now, the scalar product entering in the calculation of the ISF writes as,

$$
\begin{aligned}
\mathbf{Q} \cdot \mathbf{r}= & Q b_{\mathrm{H}}\left(\begin{array}{c}
\sin \gamma \cos \lambda \\
\sin \gamma \sin \lambda \\
\cos \gamma
\end{array}\right) \cdot\left(\begin{array}{c}
\cos \alpha \cos \eta \cos \phi-\sin \eta \sin \phi \\
\cos \alpha \sin \eta \cos \phi+\cos \eta \sin \phi \\
-\sin \alpha \cos \phi
\end{array}\right) \\
= & Q b_{\mathrm{H}}[\sin \gamma \cos \phi \cos \alpha(\cos \lambda \cos \eta+\sin \lambda \sin \eta)+\sin \gamma \sin \phi(\sin \lambda \cos \eta-\cos \lambda \sin \eta) \\
& -\cos \gamma \sin \alpha \cos \phi] \\
= & Q b_{\mathrm{H}}[\sin \gamma \cos \phi \cos \alpha \cos (\lambda-\eta)+\sin \gamma \sin \phi \sin (\lambda-\eta)-\cos \gamma \sin \alpha \cos \phi] \\
= & Q b_{\mathrm{H}}\left\{\sin \gamma\left[\cos ^{2}\left(\frac{\alpha}{2}\right) \cos (\phi-\lambda+\eta)-\sin ^{2}\left(\frac{\alpha}{2}\right) \cos (\phi+\lambda-\eta)\right]-\cos \gamma \sin \alpha \cos \phi\right\} \\
= & Q b_{\mathrm{H}} f(\alpha, \phi, \lambda, \gamma, \eta)
\end{aligned}
$$

The head group performs two independent motions:

- Uniaxial rotational diffusion of angle $\phi$ about the $\vec{l}$-axis and diffusion constant $D_{\text {head }}$ described by the Green's function:

$$
G_{\text {rot }}\left(\phi, t \mid \phi_{0}\right)=\frac{1}{2 \pi} \sum_{n=-\infty}^{\infty} \exp \left\{i n\left(\phi-\phi_{0}\right)\right\} \cdot \exp \left\{-D_{\text {head }} n^{2}|t|\right\}
$$

with the equilibrium distribution, $P_{\text {eq,rot }}(\phi)=\lim _{t \rightarrow \infty} G_{\text {rot }}\left(\phi, t \mid \phi_{0}\right)=1 /(2 \pi)$,

- Flip-flop motion of the $\vec{l}$-axis between angles $\theta=-\alpha$ and $\theta=\alpha$ and residence time $\tau_{\text {ff }}$ described by the jump diffusion with the Green's function:

$$
G_{\mathrm{ff}}\left(\theta, t \mid \theta_{0}\right)=\frac{\delta_{\theta, \alpha}}{2}\left[u \delta_{\theta_{0}, \alpha}+v \delta_{\theta_{0},-\alpha}\right]+\frac{\delta_{\theta,-\alpha}}{2}\left[u \delta_{\theta_{0},-\alpha}+v \delta_{\theta_{0}, \alpha}\right]
$$


where,

$$
\left.\begin{array}{l}
u \\
v
\end{array}\right\}=1 \pm \exp \left\{-\frac{|t|}{\tau_{\mathrm{ff}}}\right\}
$$

with the equilibrium distribution, $P_{\text {eq,ff }}(\theta)=\lim _{t \rightarrow \infty} G_{\mathrm{ff}}\left(\theta, t \mid \theta_{0}\right)=1 / 2$.

For experimental configurations where the direction of the scattering vector $\mathbf{Q}$ with respect to the membrane normal is set constant (i.e., the angle $\gamma$ is set constant for all $\lambda$ ), the ISF is calculated as,

$$
\begin{aligned}
I(Q, t)= & \sum_{\theta_{0}=-\alpha}^{\alpha} P_{\mathrm{eq}, \mathrm{ff}}\left(\theta_{0}\right) \sum_{\theta=-\alpha}^{\alpha} G_{\mathrm{ff}}\left(\theta, t \mid \theta_{0}\right) \int_{0}^{2 \pi} \frac{d \eta_{0}}{2 \pi} \int_{0}^{2 \pi} \frac{d \eta}{2 \pi} \int_{0}^{2 \pi} d \phi_{0} P_{\text {eq,rot }}\left(\phi_{0}\right) \\
& \int_{0}^{2 \pi} d \phi G_{\mathrm{rot}}\left(\phi, t \mid \phi_{0}\right) \exp \left\{i Q b_{\mathrm{H}}\left[f(\theta, \phi, \lambda, \gamma, \eta)-f\left(\theta_{0}, \phi_{0}, \lambda, \gamma, \eta_{0}\right)\right]\right\} \\
= & \sum_{\theta_{0}=-\alpha}^{\alpha} P_{\mathrm{eq}, \mathrm{ff}}\left(\theta_{0}\right) \sum_{\theta=-\alpha}^{\alpha} G_{\mathrm{ff}}\left(\theta, t \mid \theta_{0}\right) \sum_{n=-\infty}^{\infty} B_{n}(\theta, \gamma) B_{n}^{*}\left(\theta_{0}, \gamma\right) \exp \left\{-D_{\text {head }} n^{2}|t|\right\}
\end{aligned}
$$

where,

$$
B_{n}(\theta, \gamma)=\int_{0}^{2 \pi} \frac{d \eta}{2 \pi} \int_{0}^{2 \pi} \frac{d \phi}{2 \pi} \exp \{i[Q b f(\theta, \phi, \lambda, \gamma, \eta)+n \phi]\}=\frac{1}{2 \pi} \int_{0}^{2 \pi} d \eta C_{n}(\theta, \lambda, \gamma, \eta)(
$$

Using $G_{\mathrm{ff}}\left(\theta, t \mid \theta_{0}\right)$ in Eq.(A.10) back into $I(Q, t)$, we have:

$$
\begin{aligned}
I(Q, t)= & \sum_{n=-\infty}^{\infty}\left\{\frac{u}{4}\left[B_{n}(\alpha, \gamma) B_{n}^{*}(\alpha, \gamma)+B_{n}(-\alpha, \gamma) B_{n}^{*}(-\alpha, \gamma)\right]\right. \\
& \left.\frac{v}{4}\left[B_{n}(\alpha, \gamma) B_{n}^{*}(-\alpha, \gamma)+B_{n}(-\alpha, \gamma) B_{n}^{*}(\alpha, \gamma)\right]\right\} \exp \left\{-D_{\text {head }} n^{2}|t|\right\}
\end{aligned}
$$

We have,

$$
\begin{aligned}
C_{n}(\alpha, \lambda, \gamma, \eta) & =\frac{1}{2 \pi} \int_{0}^{2 \pi} d \phi \exp \left\{i\left[Q b_{\mathrm{H}} f(\alpha, \phi, \lambda, \gamma, \eta)+n \phi\right]\right\} \\
& =\frac{1}{2 \pi} \int_{0}^{2 \pi} d \phi \exp \{i n \phi\} \exp \left\{i z_{1} \cos \phi\right\} \exp \left\{i z_{2} \cos (\phi-\mu)\right\} \exp \left\{i z_{2} \cos (\phi+\mu)\right\}
\end{aligned}
$$

where,

$$
\left\{\begin{array}{l}
\mu=\lambda-\eta \\
z_{1}=-Q b_{\mathrm{H}} \cos \gamma \sin \alpha \\
z_{2}=Q b_{\mathrm{H}} \sin \gamma \cos ^{2}\left(\frac{\alpha}{2}\right) \\
z_{3}=Q b_{\mathrm{H}} \sin \gamma \sin ^{2}\left(\frac{\alpha}{2}\right)
\end{array}\right.
$$


Now, using the following relations,

$$
\left\{\begin{array}{l}
\exp \{ \pm i z \cos \psi\}=\sum_{m=-\infty}^{\infty} i^{ \pm m} \exp \{\mp i m \psi\} J_{m}(z) ; \text { Jacobi-Anger expansion } \\
\int_{0}^{2 \pi} d \phi \exp \{ \pm i k \phi\}=2 \pi \delta_{k, 0}
\end{array}\right.
$$

back into Eq;(A.15), we obtain:

$$
C_{n}(\alpha, \lambda, \gamma, \eta)=i^{n} \sum_{m=-\infty}^{\infty} \sum_{l=-\infty}^{\infty} J_{m}\left(z_{1}\right) J_{l}\left(z_{2}\right) J_{n-m-l}\left(z_{3}\right) \exp \{i(2 l+m-n) \mu\}
$$

where $J_{m}(\cdots)$ is the Bessel function of first kind and order $m$. It follows that,

$$
\begin{aligned}
B_{n}(\alpha, \gamma) & =\int_{0}^{2 \pi} \frac{d \eta}{2 \pi} C_{n}(\alpha, \lambda, \gamma, \eta)=\int_{0}^{2 \pi} \frac{d \mu}{2 \pi} C_{n}(\alpha, \lambda, \gamma, \eta) \\
& =\frac{i^{n}}{2 \pi} \sum_{m=-\infty}^{\infty} \sum_{l=-\infty}^{\infty} J_{m}\left(z_{1}\right) J_{l}\left(z_{2}\right) J_{n-m-l}\left(z_{3}\right) \int_{0}^{2 \pi} d \mu \exp \{i(2 l+m-n) \mu\} \\
& =i^{n} \sum_{l=-\infty}^{\infty} J_{n-2 l}\left(z_{1}\right) J_{l}\left(z_{2}\right) J_{l}\left(z_{3}\right) .
\end{aligned}
$$

Using the relation for the Bessel functions, $J_{n}(-x)=(-1)^{n} J_{n}(x)$, we obtain the relation: $B_{n}(\alpha, \gamma)=(-1)^{n} B_{n}(-\alpha, \gamma)$. Now, using this relation relation in Eq.(A.14), we finally obtain,

$$
\begin{aligned}
I(Q, t) & =\sum_{n=-\infty}^{\infty}\left[\frac{u+(-1)^{n} v}{2}\right]\left|B_{n}(-\alpha, \gamma)\right|^{2} \exp \left\{-D_{\text {head }} n^{2}|t|\right\} \\
& \left.=\sum_{n=-\infty}^{\infty}\left[\sum_{l=-\infty}^{+\infty} J_{n-2 l}\left(-z_{1}\right) J_{l}\left(z_{2} t\right) J_{l}\left(z_{3}\right)\right]^{2} \exp \left\{-\left[n^{2} D_{\text {head }}+\frac{1-(-1)^{n}}{2 \tau_{\text {ff }}}\right]|t| \mid\right\} .21\right)
\end{aligned}
$$

Appendix A.2. 1d Diffusion in a Harmonic Potential

Let the $z$-axis coincides with the normal to the membrane, we consider that each lipid molecule, of coordinate $z$, is undergoing as a whole a $1 \mathrm{~d}$ diffusion parallel to $z$-axis in a harmonic potential of mean force, $V(z)=k z^{2} / 2$, of force constant $k$ and relaxation time $\tau$. The Green's function, $G\left(z, t \mid z_{0}\right)$, describing such motions for the lipid molecule is given by,

$$
G\left(z, t \mid z_{0}\right)=\frac{\exp \left\{-\frac{\left(z-z_{0} \mathrm{e}^{-t / \tau}\right)^{2}}{2 \sigma_{0}^{2}\left[1-\mathrm{e}^{-2 t / \tau}\right]}\right\}}{\sqrt{2 \pi \sigma_{0}^{2}\left[1-\mathrm{e}^{-2 t / \tau}\right]}}
$$


with the equilibrium distribution of lipid molecule positions given by,

$$
P_{\mathrm{eq}}(z)=\lim _{t \rightarrow \infty} G\left(z, t \mid z_{0}\right)=\frac{\exp \left\{-\frac{z^{2}}{2 \sigma_{0}^{2}}\right\}}{\sqrt{2 \pi \sigma_{0}^{2}}}, \sigma_{0}^{2}=\frac{k_{\mathrm{B}} T}{k}
$$

Denoting by $\gamma$ the angle between the $z$-axis and the scattering $\mathbf{Q}$, the ISF can be written as,

$$
\begin{aligned}
I(Q, t) & =\left\langle e^{i \mathbf{Q} \cdot \mathbf{r}(\mathbf{t})} e^{-i \mathbf{Q} \cdot \mathbf{r}(\mathbf{0})}\right\rangle=\left\langle e^{i Q \cos \gamma z(t)} e^{-i Q \cos \gamma z(0)}\right\rangle \\
& =\int_{-\infty}^{+\infty} d z_{0} \int_{-\infty}^{+\infty} d z \mathrm{e}^{i Q \cos \gamma z} G\left(z, t \mid z_{0}\right) \mathrm{e}^{-i Q \cos \gamma z_{0}} P_{\mathrm{eq}}\left(z_{0}\right) \\
& =\int_{-\infty}^{+\infty} d z_{0} B\left(z_{0}\right) P_{\mathrm{eq}}\left(z_{0}\right)
\end{aligned}
$$

where,

$$
\left\{\begin{array}{l}
B\left(z_{0}\right)=\int_{-\infty}^{+\infty} d z \mathrm{e}^{i Q \cos \gamma z} G\left(z, t \mid z_{0}\right)=\mathrm{e}^{i y z_{0} \mathrm{e}^{-t / \tau}-(y a)^{2} / 4} \\
y=Q \cos \gamma ; a^{2}=2 \sigma_{0}^{2}\left[1-\mathrm{e}^{-2 t / \tau}\right]
\end{array}\right.
$$

Then,

$$
\begin{aligned}
I(Q, t) & =\mathrm{e}^{-(y a)^{2} / 4} \int_{-\infty}^{+\infty} d z_{0} \mathrm{e}^{-i y z_{0}\left[1-\mathrm{e}^{-t / \tau}\right]} P_{\mathrm{eq}}\left(z_{0}\right) \\
& =\exp \left\{-\frac{(y a)^{2}}{4}-\frac{\left[y \sigma_{0}\left(1-\mathrm{e}^{-t / \tau}\right)\right]^{2}}{2}\right\}
\end{aligned}
$$

Finally, we obtain:

$$
I(Q, t)=\exp \left\{-(Q \cos \gamma)^{2}\left(\frac{k_{\mathrm{B}} T}{k}\right)\left[1-\mathrm{e}^{-t / \tau}\right]\right\}
$$

\section{Appendix B. Derivation of aggregated expressions of $B$ 's amplitudes and $\Gamma$ 's rates}

Starting from a time dependent ISF, the aim of the aggregation is in general to derive an approximate expression of ISF as an expansion of only few relaxation functions, $E_{k}(Q, t)$ such that, $E_{k}(Q, t=0)=1$ and $E_{k}(Q, t \rightarrow \infty)=0$. For our purpose, we deal with single exponential relaxation function, $E_{k}(Q, t)=\exp \left\{-\Gamma_{k} t\right\}$, where $\Gamma_{k}(Q)$ is the relaxation rate. The ISF to derive an aggregation is given in the right-hand-side of Eq.(10) as,

$$
I=\left[z I_{\text {jump }} I_{\text {head }}+(1-z) I_{\text {jump }} I_{\text {tail }}\right] I_{\text {rot }} I_{\text {in-out }} I_{2 \mathrm{~d}}
$$


where $I_{\alpha}(Q, t)$ are the ISF's of local motions described in the Matryoshka model and given as $[16]$,

$$
I_{\alpha}(Q, t)=A_{\alpha}(Q)+\left[1-A_{\alpha}(Q)\right] C_{\alpha}(Q, t) ; \alpha=\text { jump, head, tail, rot, in - out, } 2 \mathrm{~d}
$$

where $A_{\alpha}(Q)$ is the amplitude and $C_{\alpha}(Q, t)$ the relaxation function such that, $C_{\alpha}(Q, t=$ $0)=1$ and $C_{\alpha}(Q, t \rightarrow \infty)=0$. As, in general, $C_{\alpha}(Q, t)$ is a multi-exponential function of time with decay rates functions of $Q$, the first step toward the aggregation is to derive a single exponential approximation of $C_{\alpha}(Q, t)$ as [16],

$$
C_{\alpha}(Q, t) \approx E_{\alpha}(Q, t)=\exp \left\{-\Gamma_{\alpha}(Q) t\right\} \text { with } \frac{1}{\Gamma_{\alpha}(Q)}=\int_{0}^{\infty} d t C_{\alpha}(Q, t)
$$

where $\Gamma_{\alpha}(Q)$ is the $Q$-dependent relaxation rate. Next, the goal is to derive the amplitudes and relaxation rates such that Eq.(B.1) can be rewritten as,

$$
\left\{\begin{array}{l}
I_{\mathrm{agg}}(Q, t)=\sum_{i=0}^{3} B_{i}(Q) E_{i}(Q, t) \\
\text { with: } i=(0=\mathrm{EISF}),(1=\text { slow }),(2=\text { intermediate }),(3=\text { fast }),
\end{array}\right.
$$

where $B_{i}(Q)$ is the amplitude and $E_{i}(Q, t)$ the relaxation function with relaxation rates such that, $\Gamma_{0}=0<\Gamma_{\text {slow }}<\Gamma_{\text {intermediate }}<\Gamma_{\text {fast }}$. To proceed, expanding Eq.(B.1) yields,

$$
\left\{\begin{aligned}
I= & {\left[z A_{\text {jump }} A_{\text {head }}+z A_{\text {jump }}\left(1-A_{\text {head }}\right) E_{\text {head }}+z\left(1-A_{\text {jump }}\right) A_{\text {head }} E_{\text {jump }}\right.} \\
& +z\left(1-A_{\text {jump }}\right)\left(1-A_{\text {head }}\right) E_{\text {jump }} E_{\text {head }}+(1-z) A_{\text {jump }} A_{\text {tail }} \\
& +(1-z) A_{\text {jump }}\left(1-A_{\text {tail }}\right) E_{\text {tail }}+(1-z)\left(1-A_{\text {jump }}\right) A_{\text {tail }} E_{\text {jump }} \\
& \left.+(1-z)\left(1-A_{\text {jump }}\right)\left(1-A_{\text {tail }}\right) E_{\text {jump }} E_{\text {tail }}\right] \times\left[A_{\text {rot }} A_{\text {in-out }} A_{2 \mathrm{~d}}\right. \\
& +\left(1-A_{\text {rot }}\right) A_{\text {in }- \text { out }} A_{2 \mathrm{~d}} E_{\text {rot }}+A_{\text {rot }}\left(1-A_{\text {in-out }}\right) A_{2 \mathrm{~d}} E_{\text {in-out }} \\
& +A_{\text {rot }} A_{\text {in-out }}\left(1-A_{2 \mathrm{~d}}\right) E_{2 \mathrm{~d}}+\left(1-A_{\text {rot }}\right)\left(1-A_{\text {in }- \text { out }}\right) A_{2 \mathrm{~d}} E_{\text {rot }} E_{\text {in-out }} \\
& +\left(1-A_{\text {rot }}\right) A_{\text {in-out }}\left(1-A_{2 \mathrm{~d}}\right) E_{\text {rot }} E_{2 \mathrm{~d}}+A_{\text {rot }}\left(1-A_{\text {in-out }}\right)\left(1-A_{2 \mathrm{~d}}\right) E_{\text {in-out }} E_{2 \mathrm{~d}} \\
& \left.+\left(1-A_{\text {rot }}\right)\left(1-A_{\text {in-out }}\right)\left(1-A_{2 \mathrm{~d}}\right) E_{\text {rot }} E_{\text {in-out }} E_{2 \mathrm{~d}}\right]
\end{aligned}\right.
$$

Next, we use the following rules,

- Hierarchy of relaxations: the hierarchy of relaxation time scales of motional processes in the Matryoshka model indicates that: $\Gamma_{\text {jump }} \sim \Gamma_{\text {tail }}>\Gamma_{\text {head }} \sim \Gamma_{\text {rot }}>\Gamma_{\text {in-out }} \sim \Gamma_{2 \mathrm{~d}}$, implying that, $E_{\text {jump }} \sim E_{\text {tail }}<E_{\text {head }} \sim E_{\text {rot }}<E_{\text {in-out }} \sim E_{2 \mathrm{~d}}$ for all $t$;

- Operation rules: for timescales $\Gamma_{i} t \sim 1$, we have:

$$
\triangleright \text { Summation: } a E_{i}+b E_{j} \approx \begin{cases}a E_{i}+b & , \quad \Gamma_{i}>\Gamma_{j} \\ a E_{i} & , \quad \Gamma_{i}<\Gamma_{j}\end{cases}
$$




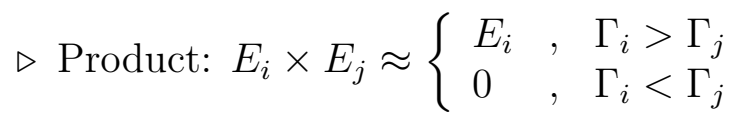

to rearrange Eq.(B.5) into 4 terms and derive the aggregated approximation in Eq.(B.4) where,

- EISF: collects all time independent terms in Eq.(B.5).

Taking the $t \rightarrow \infty$ limit (i.e., setting all $\left.E_{\alpha}(Q, t \rightarrow \infty)=0\right)$ in Eq.(B.5) gives,

$$
B_{0}=\left[z A_{\text {head }}+(1-z) A_{\text {tail }}\right] A_{\text {jump }} A_{\text {rot }} A_{\text {in-out }} A_{2 \mathrm{~d}}
$$

- Slow motions: collect all terms involving $E_{\text {in-out }}$ and $E_{2 \mathrm{~d}}$ in Eq.(B.5).

$$
\begin{aligned}
B_{\text {slow }} E_{\text {slow }}= & \left\{A_{\text {jump }} A_{\text {rot }}\left[z A_{\text {head }}+(1-z) A_{\text {tail }}\right]+T_{1}\right\}\left\{T_{2}+A_{\text {in-out }}\left(1-A_{2 \mathrm{~d}}\right) E_{2 \mathrm{~d}}\right. \\
& \left.+\left(1-A_{\text {in-out }}\right) A_{2 \mathrm{~d}} E_{\text {in-out }}+\left(1-A_{\text {in-out }}\right)\left(1-A_{2 \mathrm{~d}}\right) E_{\text {in-out }} E_{2 \mathrm{~d}}\right\}
\end{aligned}
$$

in which $T_{1}$ groups terms involving $E_{\text {jump }}, E_{\text {tail }}$ and $E_{\text {head }}$ and $T_{2}$ terms involving the products of $E_{\text {rot }}$ with $E_{\text {in-out }}$ and $E_{2 \mathrm{~d}}$ (i.e., terms $\left.\approx E_{\text {rot }}\right)$. For timescales $\Gamma_{2 \mathrm{~d}} t \sim$ $\Gamma_{\text {in-out }} t \sim 1$, both $T_{1}$ and $T_{2}$ vanish as they relax faster to zero and, therefore, will be omitted. Now, taking the $t \rightarrow 0$ limit in Eq.(B.7) (without $T_{1}$ and $T_{2}$ ) gives,

$$
B_{\text {slow }}=\left[z A_{\text {head }}+(1-z) A_{\text {tail }}\right]\left(1-A_{\text {in-out }} A_{2 \mathrm{~d}}\right) A_{\text {jump }} A_{\text {rot }} .
$$

And, taking the $t \rightarrow 0$ limit in the time derivative of Eq.(B.7) gives,

$$
\Gamma_{\text {slow }}=\frac{\left(1-A_{\text {in-out }}\right) \Gamma_{\text {in-out }}+\left(1-A_{2 \mathrm{~d}}\right) \Gamma_{2 \mathrm{~d}}}{1-A_{\text {in-out }} A_{2 \mathrm{~d}}}
$$

- Intermediate motions: collect all terms involving $E_{\text {head }}$ and $E_{\text {rot }}$ in Eq.(B.5).

For timescales $\Gamma_{\text {head }} t \sim \Gamma_{\text {rot }} t \sim 1$, we use the rules above, $E_{\text {in-out }} \sim E_{2 \mathrm{~d}} \approx 1$ and $E_{\text {jump }} \sim E_{\text {tail }} \approx 0$ to first reduces Eq.(B.5) to,

$$
\begin{aligned}
B_{\text {intermediate }} E_{\text {intermediate }}= & {\left[z A_{\text {jump }} A_{\text {head }}+z A_{\text {jump }}\left(1-A_{\text {head }}\right) E_{\text {head }}\right.} \\
& \left.+z\left(1-A_{\text {jump }}\right)\left(1-A_{\text {head }}\right) E_{\text {jump }} E_{\text {head }}+(1-z) A_{\text {jump }} A_{\text {tail }}\right] \times \\
& {\left[A_{\text {rot }}+\left(1-A_{\text {rot }}\right) A_{\text {in }- \text { out }} A_{2 \mathrm{~d}} E_{\text {rot }}+\right.} \\
& +\left(1-A_{\text {rot }}\right)\left(1-A_{\text {in-out }}\right) A_{2 \mathrm{~d}} E_{\text {rot }} E_{\text {in-out }} \\
& +\left(1-A_{\text {rot }}\right) A_{\text {in-out }}\left(1-A_{2 \mathrm{~d}}\right) E_{\text {rot }} E_{2 \mathrm{~d}} \\
& \left.+\left(1-A_{\text {rot }}\right)\left(1-A_{\text {in-out }}\right)\left(1-A_{2 \mathrm{~d}}\right) E_{\text {rot }} E_{\text {in-out }} E_{2 \mathrm{~d}}\right] \\
& - \text { time independent terms }
\end{aligned}
$$


and next, $E_{\text {rot }} E_{\text {in-out }} \approx E_{\text {rot }}, E_{\text {rot }} E_{2 \mathrm{~d}} \approx E_{\text {rot }}, E_{\text {rot }} E_{\text {in-out }} E_{2 \mathrm{~d}} \approx E_{\text {rot }}$ and $E_{\text {jump }} E_{\text {head }} \approx 0$ to,

$$
\begin{aligned}
B_{\text {intermediate }} E_{\text {intermediate }}= & \left\{z\left(1-A_{\text {head }}\right) A_{\text {rot }} E_{\text {head }}+z\left(1-A_{\text {head }}\right)\left(1-A_{\text {rot }}\right) E_{\text {head }} E_{\text {rot }}\right. \\
& \left.+\left[z A_{\text {head }}+(1-z) A_{\text {tail }}\right]\left(1-A_{\text {rot }}\right) E_{\text {rot }}\right\} A_{\text {jump }}
\end{aligned}
$$

Taking the $t \rightarrow 0$ limit in Eq.(B.11) gives,

$$
B_{\text {intermediate }}=\left\{z\left(1-A_{\text {head }} A_{\text {rot }}\right)+(1-z) A_{\text {tail }}\left(1-A_{\text {rot }}\right)\right\} A_{\text {jump }} .
$$

And, taking the $t \rightarrow 0$ limit in the time derivative of Eq.(B.11) gives,

$$
\Gamma_{\text {intermediate }}=\frac{z\left(1-A_{\text {head }}\right) \Gamma_{\text {head }}+\left[z+(1-z) A_{\text {tail }}\right]\left(1-A_{\text {rot }}\right) \Gamma_{\text {rot }}}{z\left(1-A_{\text {head }} A_{\text {rot }}\right)+(1-z) A_{\text {tail }}\left(1-A_{\text {rot }}\right)}
$$

- Fast motions: collect all terms involving $E_{\text {jump }}$ and $E_{\text {tail }}$ in Eq.(B.5).

For timescales $\Gamma_{\text {jump }} t \sim \Gamma_{\text {tail }} t \sim 1$, we use the rules above, $E_{\text {in-out }} \sim E_{2 \mathrm{~d}} \sim E_{\text {rot }} \sim$ $E_{\text {head }} \approx 1$ to reduces Eq.(B.5) to,

$$
\begin{aligned}
B_{\text {fast }} E_{\text {fast }}= & z\left(1-A_{\text {jump }}\right) A_{\text {head }} E_{\text {jump }} \\
& +z\left(1-A_{\text {jump }}\right)\left(1-A_{\text {head }}\right) E_{\text {jump }} E_{\text {head }}+(1-z) A_{\text {jump }} A_{\text {tail }} \\
& +(1-z) A_{\text {jump }}\left(1-A_{\text {tail }}\right) E_{\text {tail }}+(1-z)\left(1-A_{\text {jump }}\right) A_{\text {tail }} E_{\text {jump }} \\
& +(1-z)\left(1-A_{\text {jump }}\right)\left(1-A_{\text {tail }}\right) E_{\text {jump }} E_{\text {tail }}
\end{aligned}
$$

or,

$$
\begin{aligned}
B_{\text {fast }} E_{\text {fast }}= & {\left[z A_{\text {head }}+(1-z) A_{\text {tail }}\right]\left(1-A_{\text {jump }}\right) E_{\text {jump }}+(1-z) A_{\text {jump }}\left(1-A_{\text {tail }}\right) E_{\text {tail }} } \\
& +\left[z\left(1-A_{\text {head }}\right) E_{\text {head }}+(1-z)\left(1-A_{\text {tail }}\right) E_{\text {tail }}\right]\left(1-A_{\text {jump }}\right) E_{\text {jump }}
\end{aligned}
$$

Taking the $t \rightarrow 0$ limit in Eq.(B.15) gives,

$B_{\text {fast }}=\left(1-A_{\text {jump }}\right)+(1-z) A_{\text {jump }}\left(1-A_{\text {tail }}\right)=z\left(1-A_{\text {jump }}\right)+(1-z)\left(1-A_{\text {jump }} A_{\text {tail }}(\right.$

And, taking the $t \rightarrow 0$ limit in the time derivative of Eq.(B.15) gives,

$$
\Gamma_{\text {fast }}=\frac{\left(1-A_{\text {jump }}\right) \Gamma_{\text {jump }}+(1-z)\left(1-A_{\text {tail }}\right) \Gamma_{\text {tail }}}{B_{\text {fast }}}
$$

\section{Appendix C. Relation between experimental $A$ 's and theoretical $B$ 's amplitudes}

Given that the theoretical aggregated ISF in Eq.(B.4) writes as follows,

$$
I_{\text {agg }}(Q, t)=\sum_{i=0}^{n} B_{i}(Q) E_{i}(Q, t) ; \sum_{i=0}^{n} B_{i}(Q)=1 ; B_{i}(Q=0)=\delta_{i, 0},
$$


we assume that the experimental ISF can also be written in the same way as,

$$
I_{\exp }(Q, t)=\sum_{i=0}^{n} A_{i}(Q) E_{i}(Q, t) ; \quad \sum_{i=0}^{n} A_{i}(Q)=1,
$$

with the same $E_{i}(Q, t)$ but different amplitudes $A_{i}(Q)$ and such that, $0<A_{i}(Q=0)<1, \forall i$. The correspondence between $I_{\mathrm{agg}}(Q, t)$ and $I_{\exp }(Q, t)$ can be written as,

$$
I_{\exp }(Q, t)=m I_{\text {agg }}(Q, t)+\underbrace{\sum_{i=0}^{n} \varepsilon_{i}(Q) E_{i}(Q, t)}_{\text {error terms }},
$$

where $m$ is the fraction of observable mobile H-atoms and $\varepsilon_{i}(Q)$ 's the errors accounting for the fraction of immobile $\mathrm{H}$-atoms and other experimental errors like multiple scatterings, etc. Collecting in Eq.(C.3) terms under the same relaxation function $E_{i}(Q, t)$, we obtain the general relationship between $B(Q)$ 's and $A(Q)$ 's as,

$$
A_{i}(Q)=m B_{i}(Q)+\varepsilon_{i}(Q) \quad ; \quad \forall i
$$

From the point of view of the analysis of experimental data, the expression in Eq.(C.4) involves $n+2$ unknowns to determine: $m$ and $n+1$ functions $\varepsilon_{i}(Q)$. Therefore, in the absence of any information we use the closure relation (obtained by construction) satisfied by the unknowns and assume that the errors of the quasi-elastic terms $(i>0)$ are all identical, i.e.,

$$
\left\{\begin{array}{l}
m+\sum_{i=0}^{n} \varepsilon_{i}(Q)=1 \\
\varepsilon_{i}(Q)=\varepsilon(Q) \quad ; i>0
\end{array} \Longrightarrow \varepsilon_{i}(Q)=\frac{\left[1-m-\varepsilon_{0}(Q)\right]}{n} ; i>0,\right.
$$

thus reducing the number of unknowns from $n+2$ to 2 . Using this back in Eq.(C.4), we obtain,

$$
\left\{\begin{array}{l}
A_{0}(Q)=m B_{0}(Q)+\varepsilon_{0}(Q) \\
A_{i}(Q)=m B_{i}(Q)+\frac{\left[1-m-\varepsilon_{0}(Q)\right]}{n} ; i>0
\end{array}\right.
$$

where the remaining unknowns are $m$ and $\varepsilon_{0}(Q)$.

\section{References}

[1] M. Luckey. Membrane structural biology: with biochemical and biophysical foundations. Cambridge University Press, New York, 2008.

[2] R. Lipowsky and L. Sackmann. Structure and Dynamics of Membranes. North Holland, 1995. 
[3] S. König, T. M. Bayerl, G. Coddens, D. Richter, and E. Sackmann. Hydration dependence of chain dynamics and local diffusion in l-alphadipalmitoylphosphtidylcholine multilayers studied by incoherent quasielastic neutron scattering. Biophys. J., 68:1871-1880, 1995.

[4] E. Lindahl and O. Edholm. Mesoscopic undulations and thickness fluctuations in lipid bilayers from molecular dynamics simulations. Biophys. J., 79:426-433, 2000.

[5] W. Pfeiffer, Th. Henkel, E. Sackmann, W. Knoll, and D. Richter. Local dynamics of lipid bilayers studied by incoherent quasi-elastic neutron scattering. Europhys. Lett., 8(2):201-206, 1989. URL https: //doi .org/10.1209/0295-5075/8/2/016.

[6] U. Wanderlingh, G. DAngelo, V. Conti Nibali, M. Gonzalez, C. Crupi, and C. Mondelli. Influence of gramicidin on the dynamics of dmpc studied by incoherent elastic neutron scattering. . Phys.: Condens. Matter, 20:104214, 2008.

[7] M. C. Rheinstädter, C. Ollinger, G. Fragneto, F. Demmel, and T. Salditt. Collective dynamics of lipid membranes studied by inelastic neutron scattering. Phys. Rev. Lett., 93:108107, 2004.

[8] F. Natali, C. Castellano, D. Pozzi, and A. Congiu Castellano. Dynamic properties of an oriented lipid/DNA complex studied by neutron scattering. Biophysical Journal, 88(2):1081-1090, February 2005. doi: 10.1529/biophysj.104.042788. URL https://doi.org/10.1529/biophysj.104.042788.

[9] M. Trapp, T. Gutberlet, F. Juranyi, T. Unruh, B. Demé, M. Tehei, and J. Peters. Hydration dependent studies of highly aligned multilayer lipid membranes by neutron scattering. J. Chem. Phys., 133:164505, 2010.

[10] S. Gupta and G. J. Schneider. Modeling the dynamics of phospholipids in the fluid phase of liposomes. Soft Matter, 16:3245-3256, 2020.

[11] S. König, W. Pfeiffer, T. Bayerl, D. Richter, and E. Sackmann. Molecular dynamics of lipid bilayers studied by incoherent quasi-elastic neutron scattering. J. Phys. II France, 2:1589-1615, 1992. URL https://doi.org/10.1051/jp2:1992100.

[12] U. Wanderlingh, G. DAngelo, C. Branca, V. Conti Nibali, A. Trimarchi, S. Rifici, D. Finocchiaro, C. Crupi, J. Ollivier, and H. D. Middendorf. Multi-component modeling of quasielastic neutron scattering from phospholipid membranes. J. Chem. Phys., 140:174901, 2014. URL https://doi.org/10. $1063 / 1.4872167$.

[13] Q. Berrod, K. Lagrené, J. Ollivier, and J.-M. Zanotti. Inelastic and quasi-elastic neutron scattering. application to soft-matter. EPJ Web of Conferences, 188:05001, 2018. URL https://doi.org/10. 1051 /epjconf/201818805001.

[14] D. J. Bicout. Diffusion incohrente des neutrons : modles analytiques pour la dynamique interne des protines. J. Phys. IV France, 130:115-132, 2005. URL https://doi.org/10.1051/jp4:2005130008.

[15] L. Carpentier, M. Be, A. M. Giroud-Godquin, P. Maldivi, and J. C. Marchon. Alkyl chain motions in columnar mesophases: A quasielastic neutron scattering study of dicopper tetrapalmitate. Mol. Phys., 68(6):1367-1378, 1989 .

[16] D. J. Bicout. Incoherent neutron scattering functions for diffusion inside two concentric spheres. Phys. Rev. E, 62:261-271, Jul 2000. doi: 10.1103/PhysRevE.62.261. URL https://link.aps.org/doi/10. 1103/PhysRevE. 62.261.

[17] In6: Cold neutron time-focusing time-of-flight spectrometer. URL http://www. ill.eu/instruments-support/instruments-groups/instruments/in6/description/ instrument-layout/.

[18] J. Peters, J. Marion, F. Natali, E. Kats, and D. J. Bicout. The dynamical transition of lipid multilamellar bilayers as a matter of cooperativity. J. Phys. Chem. B, 121(28):6860-6868, 2017. doi: 10.1021/acs.jpcb.7b05167. URL https://doi.org/10.1021/acs.jpcb.7b05167.

[19] D. Richard, M. Ferrand, and G. J. Kearley. Analysis and visualisation of neutron-scattering data. Journal of Neutron Research, 4(1):33-39, December 1996. doi: 10.1080/10238169608200065. URL https://doi.org/10.1080/10238169608200065.

[20] Matthew Newville, Till Stensitzki, Daniel B. Allen, Antonino Ingargiola, and Andrew Nelson. Lmfit: Non-linear least-square minimization and curve-fitting for python. June 2016. URL https://ui. adsabs. harvard. edu/abs/2016ascl. soft06014N. 
[21] A. Cissé and al. In preparation. 2021.

[22] T. Matsuo and al. In preparation. 2021.

[23] Computational chemistry comparison and benchmark database, Release 21, August 2020. URL https: //cccbdb.nist.gov/.

[24] Wiebke Knoll. Le Rôle de la myéline dans les maladies dégénératives. Theses, Université de Grenoble, September 2012. URL https://tel.archives-ouvertes.fr/tel-00744455.

[25] Avanti polar lipids website, Copyright 2021 Croda International Plc. URL avantilipids.com.

[26] Stephanie Tristram-Nagle, Yufeng Liu, Justin Legleiter, and John F. Nagle. Structure of gel phase DMPC determined by x-ray diffraction. Biophysical Journal, 83(6):3324-3335, December 2002. doi: 10.1016/s0006-3495(02)75333-2. URL https://doi .org/10.1016/s0006-3495(02)75333-2.

[27] Anthony Watts, Karl Harlos, and Derek Marsh. Charge-induced tilt in ordered-phase phosphatidylglycerol bilayers evidence from x-ray diffraction. Biochimica et Biophysica Acta (BBA) - Biomembranes, 645(1):91-96, June 1981. doi: 10.1016/0005-2736(81)90515-0. URL https://doi.org/10 . 1016/0005-2736 (81)90515-0.

[28] Bachir Aoun, Eric Pellegrini, Marcus Trapp, Francesca Natali, Laura Cantù, Paola Brocca, Yuri Gerelli, Bruno Demé, Michael Marek Koza, Mark Johnson, and Judith Peters. Direct comparison of elastic incoherent neutron scattering experiments with molecular dynamics simulations of dmpc phase transitions. The European Physical Journal E, 39(4), apr 2016. doi: 10.1140/epje/i2016-16048-y. URL https://doi.org/10.1140/epje/i2016-16048-y.

[29] Wiebke Knoll, Judith Peters, Petri Kursula, Yuri Gerelli, Jacques Ollivier, Bruno Demé, Mark Telling, Ewout Kemner, and Francesca Natali. Structural and dynamical properties of reconstituted myelin sheaths in the presence of myelin proteins MBP and p2 studied by neutron scattering. Soft Matter, 10 (3):519-529, 2014. doi: 10.1039/c3sm51393a. URL https ://doi.org/10.1039/c3sm51393a.

[30] A. J. Dianoux, F. Volino, and H. Hervet. Incoherent scattering law for neutron quasi-elastic scattering in liquid crystals. Mol. Phys., 30(4):1181-1194, 1975.

[31] A. J. Dianoux, M. Pineri, and F. Volino. Incoherent scattering law for restricted diffusion inside a volume with an anisotropic shape: Application to the problem of water absorbed in nafion membranes. Mol. Phys., 46(1):129-137, 1982.

[32] M. Abramowitz and I. A. Stegun. Handbook of Mathematical Functions. Dover, New York, 1972. 